\title{
Bottomonium spectrum revisited
}

\author{
Jorge Segovia* \\ Physik-Department, Technische Universität München, James-Franck-Strasse 1, 85748 Garching, Germany \\ and Instituto Universitario de Física Fundamental y Matemáticas (IUFFyM), \\ Universidad de Salamanca, E-37008 Salamanca, Spain \\ Pablo G. Ortega ${ }^{\dagger}$ \\ CERN (European Organization for Nuclear Research), CH-1211 Geneva, Switzerland
}

\author{
David R. Entem ${ }^{\ddagger}$ and Francisco Fernández ${ }^{\S}$ \\ Grupo de Física Nuclear and Instituto Universitario de Física Fundamental y Matemáticas (IUFFyM), \\ Universidad de Salamanca, E-37008 Salamanca, Spain \\ (Received 21 January 2016; published 20 April 2016)
}

\begin{abstract}
We revisit the bottomonium spectrum motivated by the recently exciting experimental progress in the observation of new bottomonium states, both conventional and unconventional. Our framework is a nonrelativistic constituent quark model which has been applied to a wide range of hadronic observables from the light to the heavy quark sector, and thus the model parameters are completely constrained. Beyond the spectrum, we provide a large number of electromagnetic, strong and hadronic decays in order to discuss the quark content of the bottomonium states and give more insights about a better way to determine their properties experimentally.
\end{abstract}

DOI: 10.1103/PhysRevD.93.074027

\section{INTRODUCTION}

\section{A. Experimental situation}

Bottomonium, a bound system of a bottom $(b)$ quark and its antiquark $(\bar{b})$, was discovered as spin-triplet states called $\Upsilon(1 S), \Upsilon(2 S)$ and $\Upsilon(3 S)$ by the E288 Collaboration at Fermilab in 1977 in proton scattering on $C u$ and $P b$ targets studying muon pairs in a regime of invariant masses larger than $5 \mathrm{GeV}[1,2]$. Later, they were better studied at various $e^{+} e^{-}$storage rings. The two triplet $P$-wave states $\chi_{b J}(2 P)$ and $\chi_{b J}(1 P)$ with $J=0,1,2$ were discovered in radiative decays of the $\Upsilon(3 S)$ and $\Upsilon(2 S)$ in 1982 [3,4] and 1983 $[5,6]$, respectively.

Despite such early measurements, during the next 30 years there were no significant contributions to the spectrum of bottomonium. Only the radial excitations of the vector bottomonium family $\Upsilon(4 S), \Upsilon(10860)$, and $\Upsilon(11020)$ were observed $[7,8]$. This was largely because the $B$ factories were not usually considered ideal facilities for the study of the bottomonium spectrum since their energy was tuned to the peak of the $\Upsilon(4 S)$

\footnotetext{
*jorge.segovia@tum.de

pgarciao@cern.ch

entem@usal.es

$\S_{\text {fdz@usal.es }}$
}

Published by the American Physical Society under the terms of the Creative Commons Attribution 3.0 License. Further distribution of this work must maintain attribution to the author(s) and the published article's title, journal citation, and DOI. resonance, which decays in almost $100 \%$ of cases to a $B \bar{B}$ pair.

The situation has changed dramatically in the last few years with many bottomonium states observed. In 2008, the spin-singlet pseudoscalar partner $\eta_{b}(1 S)$ was found by the $B A B A R$ Collaboration with a mass of $9388.9_{-2.3}^{+3.1} \pm$ $2.7 \mathrm{MeV}$ [9]. A second measurement of BABAR found a figure slightly higher $9394_{-4.9}^{+4.8} \mathrm{MeV}$ but perfectly compatible. A later measurement of the CLEO [10] Collaboration gave a value of $9391 \pm 6.6 \mathrm{MeV}$. The Belle Collaboration [11], with a simultaneous fit of its mass and width, obtains a value of $m_{\eta_{b}(1 S)}=(9402.4 \pm 1.5 \pm 1.8) \mathrm{MeV}$ for the mass and $\Gamma_{\eta_{b}(1 S)}=\left(10.8_{-3.7-2.0}^{+4.0+4.5}\right) \mathrm{MeV}$ for the width. This is the most precise measurement of the $\eta_{b}(1 S)$ mass and furthermore provides its total decay width.

In Ref. [12] the BABAR Collaboration searched for radiative decays to the $\eta_{b}(1 S)$ and $\eta_{b}(2 S)$ states. Despite their results being largely inconclusive, they observed a signal of the $\eta_{b}(2 S)$ state with a mass over a range of approximately $9974<m_{\eta_{b}(2 S)}<10015 \mathrm{MeV}$. Then, the CLEO Collaboration presented evidence for the first successful observation of $\eta_{b}(2 S)$ in $\Upsilon(2 S) \rightarrow \eta_{b}(2 S) \gamma$ decays at a mass of $9974.6 \pm 2.3 \pm 2.1 \mathrm{MeV}$ [13]. And soon after that, the Belle Collaboration [11] reported a signal for the $\eta_{b}(2 S)$ using the $h_{b}(2 P) \rightarrow \eta_{b}(2 S) \gamma$ transition at a mass of $\left(9999.0 \pm 3.5_{-1.9}^{+2.8}\right) \mathrm{MeV}$. This value is clearly incompatible with the previous one. An analysis performed by Belle [14] with almost 17 times more data found no evidence for a signal in the energy 
region around $9975 \mathrm{MeV}$, casting doubt on the CLEO result.

Experimentalists have been only able to distinguish the $\Upsilon\left(1^{3} D_{2}\right)$ state of the triplet $\Upsilon\left(1^{3} D_{J}\right)$ [15,16]. In Ref. [16] the $J=2$ member of the $\Upsilon\left(1^{3} D_{J}\right)$ spin-triplet was observed through the $\Upsilon(3 S) \rightarrow \gamma \gamma \Upsilon\left(1^{3} D_{J}\right) \rightarrow$ $\gamma \gamma \pi^{+} \pi^{-} \Upsilon(1 S)$ decay chain with a significance of 5.8 standard deviations including systematic uncertainties. For the other two members of this spin-triplet, $\Upsilon\left(1^{3} D_{1}\right)$ and $\Upsilon\left(1^{3} D_{3}\right)$, the significances were much lower, 1.8 and 1.6 respectively, and thus no experimental observation can be claimed.

Evidence for the lowest spin-singlet $P$-wave state, $h_{b}(1 P)$, was first reported by the BABAR Collaboration in the transition $\Upsilon(3 S) \rightarrow \pi^{0} h_{b}(1 P) \rightarrow \pi^{0} \gamma \eta_{b}(1 S)$ [17]. They found a $3 \sigma$ excess of events in the recoil mass distribution against $\pi^{0}$ at a mass of $(9902 \pm 4 \pm 2) \mathrm{MeV}$. This spin-singlet $P$-wave state is expected to be very close in mass to the spin-weighted average of the triplet states $\left\langle m\left(1^{3} P_{J}\right)\right\rangle=9899.9 \mathrm{MeV}$. The first significant signal for this state come from the Belle Collaboration in the $\Upsilon(5 S) \rightarrow h_{b}(1 P) \pi^{+} \pi^{-}$transition [18]. They were also able to distinguish its first radial excitation, $h_{b}(2 P)$. The measured masses of the $h_{b}(1 P)$ and $h_{b}(2 P)$ states were $9898.25 \pm 1.06_{-1.07}^{+1.03} \mathrm{MeV}$ and $10259.76 \pm 0.64_{-1.03}^{+1.43} \mathrm{MeV}$.

The proton-(anti)proton colliders have joined recently in the search of bottomonium states. A clear example is the observation of the $\chi_{b J}(n P)$ states produced in protonproton collisions at the LHC at $\sqrt{s}=7 \mathrm{TeV}$ and recorded by the ATLAS detector [19]. These states have been reconstructed through their radiative decays to $\Upsilon(1 S, 2 S)$ with $\Upsilon \rightarrow \mu^{+} \mu^{-}$. In addition to the mass peaks corresponding to the decay modes $\chi_{b J}(1 P, 2 P) \rightarrow \Upsilon(1 S) \gamma$, a new structure centered at a mass of $10.530 \pm 0.005 \pm$ $0.009 \mathrm{GeV}$ has been also observed, in both the $\Upsilon(1 S) \gamma$ and $\Upsilon(2 S) \gamma$ decay modes. This structure has been assigned to the $\chi_{b J}(3 P)$ system. Soon after that, the D0 Collaboration observed a peak in the $\Upsilon(1 S) \gamma$ final state at a mass of $10.551 \pm 0.014 \pm 0.017 \mathrm{GeV}$ [20] which is compatible with the new state observed by the ATLAS Collaboration. The LHCb Collaboration has recently determined the mass of the $\chi_{b 1}(3 P)$ to be $m\left(\chi_{b 1}(3 P)\right)=$ $10515_{-3.9}^{+2.2}$ (stat) $)_{-2.1}^{+1.5}$ (syst) $\mathrm{MeV}[21]$.

We have discussed in this section the whole spectrum of bottomonium reported in 2014 by the Particle Data Group (PDG) [22] except two states: the $X(10610)^{ \pm}$and $X(10650)^{ \pm}$. These two states were observed by the Belle Collaboration [23] in the mass spectra of the $\pi^{ \pm} \Upsilon(n S)(n=1,2,3)$ and $\pi^{ \pm} h_{b}(m P)(m=1,2)$ pairs that are produced in association with a single charged pion in $\Upsilon(5 S)$ decays. The measured masses and widths of the two structures averaged over the five final states are $(10607.2 \pm 2.0) \mathrm{MeV}$ and $(18.4 \pm 2.4) \mathrm{MeV}$ for the
$X(10610)^{ \pm}$, and $(10652.2 \pm 1.5) \mathrm{MeV}$ and $(11.5 \pm$ 2.2) $\mathrm{MeV}$ for $X(10650)^{ \pm}$. Their large mass, indicating the presence of two bottom quarks in their composition, together with their possession of electrical charge, marks these states as necessarily unconventional. Their proximity to the $B B^{*}$ and $B^{*} B^{*}$ thresholds makes their identification as molecular states an attractive possibility. However, there is a strong discussion within the scientific community about other possible interpretations [24-27].

\section{B. Theoretical tool(s)}

The description of hadrons containing two heavy quarks is a rather challenging problem from the point of view of QCD. One has to add the complications of a nonperturbative low-energy dynamics to those usually coming from solving the bound state problem in quantum field theory.

A proper relativistic quantum field theoretical treatment of the heavy quarkonium system based on the BetheSalpeter equation has proved to be difficult despite its relative success in the last few years [28-30].

The two most promising approaches to the bottomonium bound state problem are Effective Field Theories (EFTs) and lattice gauge theories. EFTs directly derived from QCD, like nonrelativistic QCD (NRQCD) [31,32] or potential nonrelativistic QCD (pNRQCD) [33,34] (for some reviews see Refs. [35,36]), disentangle the dynamics of the heavy quarks from the dynamics of the light degrees of freedom efficiently and in a model-independent way. The fully relativistic dynamics can, in principle, be treated without approximations in lattice gauge theories (see, for instance, Ref. [37] for a standard lattice heavy quarkonium spectrum). Heavy quark calculations within these two approaches have experienced a considerable progress for states away from threshold. However, the threshold regions remain troublesome for the EFTs as well as latticeregularized QCD [38]. Moreover, the lattice calculations of excited states have been only recently pioneered and the full treatment of bottomonium on the lattice seems to be tricky (for a global picture on lattice-regularized QCD calculations and their complexities in the bottomonium sector, the reader is referred to [39-45] and references therein).

All this together explains why many of our expectations in heavy quarkonia still rely on potential models. Potential formulations have been successful at describing the heavy quark-antiquark system since the early days of charmonium (see e.g. [46-60]). Moreover, the predictions within this formalism of heavy quarkonium properties related with decays and reactions have turned to be very valuable for experimental searches. One can mention, for instance, the remarkable success of the ${ }^{3} P_{0}$ strong decay model [61-67]. Finally, the easy way to extend the quark model for describing multiquark systems makes this framework a suitable one for exploratory purposes. The results presented 
herein are based on a derived version of this approach: a nonrelativistic constituent quark model (CQM).

Spontaneous chiral symmetry breaking of the QCD Lagrangian together with the perturbative one-gluon exchange (OGE) and the nonperturbative confining interaction are the main pieces of constituent quark models. Using this idea, Vijande et al. [68] developed a model of the quark-antiquark interaction which is able to describe meson phenomenology from the light to the heavy quark sector. We have adopted this model and fine tune its parameters to reproduce the highest excited states that appear in the light quark sector [69]. The reason for that lies in the fact that it is widely believed that confinement is flavor independent. Therefore, the interactions which largely determine the high energy spectrum of heavy quarkonia should be constrained also by the light quark sector.

The quark model parameters relevant for this work are shown in Table I. In the heavy quark sector chiral symmetry is explicitly broken and Goldstone-boson exchanges do not appear. Thus, OGE and confinement are the only interactions remaining. Explicit expressions of these interactions and a brief description of the potential is given in Appendix A. Further details about the quark model and the fine-tuned model parameters can be found in Refs. [68-70].

Masses of meson states are a relevant piece of information about their structure. However, a more complete description can be achieved studying mesonic decays. In this way, we are checking particular regions of the wave function and not an average over the all meson size as in the calculation of the mass spectrum. Appendix B provides the necessary formulation to carry out the calculations presented herein on annihilation processes and on electromagnetic, strong and hadronic decays.

Two sections of this manuscript are still to be introduced. In Sec. II we discuss our quark model results and compare them with the available experimental data. We finish summarizing and giving some conclusions in Sec. III.

TABLE I. Quark model parameters.

\begin{tabular}{lcc}
\hline \hline Quark masses & $m_{n}(\mathrm{MeV})$ & 313 \\
& $m_{s}(\mathrm{MeV})$ & 555 \\
& $m_{b}(\mathrm{MeV})$ & 5110 \\
OGE & $\hat{r}_{0}(\mathrm{fm})$ & 0.181 \\
& $\hat{r}_{g}(\mathrm{fm})$ & 0.259 \\
& $\alpha_{0}$ & 2.118 \\
& $\Lambda_{0}\left(\mathrm{fm}^{-1}\right)$ & 0.113 \\
Confinement & $\mu_{0}(\mathrm{MeV})$ & 36.976 \\
& $a_{c}(\mathrm{MeV})$ & 507.4 \\
& $\mu_{c}\left(\mathrm{fm}^{-1}\right)$ & 0.576 \\
& $\Delta(\mathrm{MeV})$ & 184.432 \\
& $a_{s}$ & 0.81 \\
\hline \hline
\end{tabular}

\section{RESULTS AND DISCUSSION}

Table II shows the bottomonium spectrum (up to spin $J=3$ ) predicted by our constituent quark model. All world average masses reported in the PDG [22] are also shown.

TABLE II. Masses, in $\mathrm{MeV}$, of bottomonium states (up to spin $J=3$ ) predicted by our constituent quark model.

\begin{tabular}{|c|c|c|c|c|c|}
\hline State & $J^{P C}$ & $n L$ & Theory $(\mathrm{MeV})$ & Experiment $(\mathrm{MeV})$ & [22] \\
\hline \multirow[t]{3}{*}{$\eta_{b}$} & $0^{-+}$ & $1 S$ & 9455 & $9398.0 \pm 3.2$ & \\
\hline & & $2 S$ & 9990 & $9999.0 \pm 3.5_{-1.9}^{+2.8}$ & \\
\hline & & $3 S$ & 10330 & $\ldots$ & \\
\hline \multirow[t]{3}{*}{$\chi_{b 0}$} & $0^{++}$ & $1 P$ & 9855 & $9859.44 \pm 0.42 \pm 0.31$ & \\
\hline & & $2 P$ & 10221 & $10232.5 \pm 0.4 \pm 0.5$ & \\
\hline & & $3 P$ & 10500 & $\ldots$ & \\
\hline \multirow[t]{3}{*}{$h_{b}$} & $1^{+-}$ & $1 P$ & 9879 & $9899.3 \pm 1.0$ & \\
\hline & & $2 P$ & 10240 & $10259.8 \pm 0.5 \pm 1.1$ & \\
\hline & & $3 P$ & 10516 & $\ldots$ & \\
\hline \multirow[t]{11}{*}{$\Upsilon$} & $1^{--}$ & $1 S$ & 9502 & $9460.30 \pm 0.26$ & \\
\hline & & $2 S$ & 10015 & $10023.26 \pm 0.31$ & \\
\hline & & $1 D$ & 10117 & $\ldots$ & \\
\hline & & $3 S$ & 10349 & $10355.2 \pm 0.5$ & \\
\hline & & $2 D$ & 10414 & $\cdots$ & \\
\hline & & $4 S$ & 10607 & $10579.4 \pm 1.2$ & \\
\hline & & $3 D$ & 10653 & $\ldots$ & \\
\hline & & $5 S$ & 10818 & $10876 \pm 11$ & \\
\hline & & $4 D$ & 10853 & $\ldots$ & \\
\hline & & $6 S$ & 10995 & $11019 \pm 8$ & \\
\hline & & $5 D$ & 11023 & $\ldots$ & \\
\hline \multirow[t]{3}{*}{$\chi_{b 1}$} & $1^{++}$ & $1 P$ & 9874 & $9892.78 \pm 0.26 \pm 0.31$ & \\
\hline & & $2 P$ & 10236 & $10255.46 \pm 0.22 \pm 0.50$ & \\
\hline & & $3 P$ & 10513 & $10515.7_{-3.9-2.1}^{+2.2+1.5}$ & [21] \\
\hline \multirow[t]{3}{*}{$\eta_{b 2}$} & $2^{-+}$ & $1 D$ & 10123 & $\ldots$ & \\
\hline & & $2 D$ & 10419 & $\cdots$ & \\
\hline & & $3 D$ & 10658 & $\cdots$ & \\
\hline \multirow[t]{7}{*}{$\chi_{b 2}$} & $2^{++}$ & $1 P$ & 9886 & $9912.21 \pm 0.26 \pm 0.31$ & \\
\hline & & $2 P$ & 10246 & $10268.65 \pm 0.22 \pm 0.50$ & \\
\hline & & $1 F$ & 10315 & $\ldots$ & \\
\hline & & $3 P$ & 10521 & $\cdots$ & \\
\hline & & $2 F$ & 10569 & $\cdots$ & \\
\hline & & $4 P$ & 10744 & $\cdots$ & \\
\hline & & $3 F$ & 10782 & $\cdots$ & \\
\hline \multirow[t]{3}{*}{$\Upsilon_{2}$} & $2^{--}$ & $1 D$ & 10122 & $10163.7 \pm 1.4$ & \\
\hline & & $2 D$ & 10418 & $\cdots$ & \\
\hline & & $3 D$ & 10657 & $\cdots$ & \\
\hline \multirow[t]{3}{*}{$h_{b 3}$} & $3^{+-}$ & $1 F$ & 10322 & $\cdots$ & \\
\hline & & $2 F$ & 10573 & $\cdots$ & \\
\hline & & $3 F$ & 10785 & $\cdots$ & \\
\hline \multirow[t]{7}{*}{$\Upsilon_{3}$} & $3^{--}$ & $1 D$ & 10127 & $\cdots$ & \\
\hline & & $2 D$ & 10422 & $\cdots$ & \\
\hline & & $1 G$ & 10506 & $\ldots$ & \\
\hline & & $3 D$ & 10660 & $\ldots$ & \\
\hline & & $2 G$ & 10712 & $\ldots$ & \\
\hline & & $4 D$ & 10860 & $\ldots$ & \\
\hline & & $3 G$ & 10904 & $\ldots$ & \\
\hline \multirow[t]{3}{*}{$\chi_{b 3}$} & $3^{++}$ & $1 F$ & 10321 & $\ldots$ & \\
\hline & & $2 F$ & 10573 & $\ldots$ & \\
\hline & & $3 F$ & 10785 & $\ldots$ & \\
\hline
\end{tabular}


The masses for those states that are not yet considered as well established by PDG have been taken from the original experimental works. It is inferred from Table II that a global description of the bottomonium spectrum is obtained by our CQM.

A detailed discussion about the particular features of our spectrum will be given in the following subsections. They are complemented by computing the decay properties of the studied mesons. A comment on the theoretical errors of our results is due here. The issue of determining theoretical errors in quark models is an unresolved shortcoming of the approach. The reason for this lies on the way to fix the model parameters. The set of parameters are fitted to reproduce a certain number of hadron observables within a determinate range of agreement with experiment. Therefore, it is difficult to assign an error to those parameters and, as a consequence, to the magnitudes calculated when using them. As the range of agreement between theory and experiment is around $10-20 \%$, this value can be taken as an estimation of the errors of our results.

\section{A. The $\eta_{b}$ states}

Table II shows the predicted masses of the $\eta_{b}$ states. The hyperfine mass splitting of singlet-triplet states, i.e. $\Delta m_{h f}\left[\eta_{b}(n S)\right]=m\left(n^{3} S_{1}\right)-m\left(n^{1} S_{0}\right)$, probes the spin dependence of bound-state energy levels and imposes constraints on theoretical descriptions. For the ground states, $n=1$, it is given experimentally by [22]

$$
\Delta m_{h f}\left[\eta_{b}(1 S)\right]=62.3 \pm 3.2 \mathrm{MeV},
$$

which is higher than the theoretical prediction of EFTs, $41 \pm 11_{-8}^{+9}$ [71], and compatible with the lattice regularized QCD result, $(60.3 \pm 7.7) \mathrm{MeV}$ [42]. The Belle Collaboration, with a simultaneous fit of the mass and width of the $\eta_{b}(1 S)$, reduces this hyperfine splitting and obtains a value of $(57.9 \pm 2.3) \mathrm{MeV}$ [11]. The hyperfine mass splitting predicted by our quark model is $47 \mathrm{MeV}$, higher than the result obtained by EFTs but still lower than the experimental data and lattice regularized QCD computation.

We predict for the $\eta_{b}(2 S)$ state a mass of $9990 \mathrm{MeV}$ which is in good agreement with the last experimental measurement performed by the Belle Collaboration [11], $\left(9999.0 \pm 3.5_{-1.9}^{+2.8}\right) \mathrm{MeV}$. The corresponding theoretical hyperfine mass splitting $\Delta m_{h f}\left[\eta_{b}(2 S)\right]=25 \mathrm{MeV}$ is in excellent agreement with the experimental one $\Delta m_{h f}\left[\eta_{b}(2 S)\right]=24.3_{-4.5}^{+4.0} \mathrm{MeV}$ [11]. It is worth mentioning that our splitting is also in very good agreement with the latest results of lattice regularized QCD, $\Delta m_{h f}\left[\eta_{b}(2 S)\right]=$ (23.5-28.0) MeV [42].

One can see in Table II that our predicted mass for the $\eta_{b}(3 S)$ is $10330 \mathrm{MeV}$. Its corresponding hyperfine mass
TABLE III. Decay widths and branching fractions of annihilation rates, radiative decays and hadronic transitions for the $\eta_{b}$ states. There is no experimental data available.

\begin{tabular}{lccc}
\hline \hline Initial state & Final state & $\Gamma_{\text {The }}(\mathrm{keV})$ & $\mathcal{B}_{\text {The }}\left(10^{-2}\right)$ \\
\hline$\eta_{b}(1 S)$ & $g g$ & $20.18 \mathrm{MeV}$ & $\sim 100.00$ \\
& $\gamma \gamma$ & 0.69 & $3.42 \times 10^{-3}$ \\
$\eta_{b}(2 S)$ & total & $20.18 \mathrm{MeV}$ & 100.00 \\
& $g g$ & $10.64 \mathrm{MeV}$ & 99.86 \\
& $\gamma \gamma$ & 0.36 & $3.38 \times 10^{-3}$ \\
& $\gamma h_{b}(1 P)$ & 2.85 & $2.68 \times 10^{-2}$ \\
& $\gamma \Upsilon(1 S)$ & $4.50 \times 10^{-2}$ & $4.22 \times 10^{-4}$ \\
& $\pi \pi \eta_{b}(1 S)$ & 11.27 & $10.58 \times 10^{-2}$ \\
& total & $10.66 \mathrm{MeV}$ & 100.00 \\
$\eta_{b}(3 S)$ & $g g$ & $7.94 \mathrm{MeV}$ & 99.93 \\
& $\gamma \gamma$ & 0.27 & $3.40 \times 10^{-3}$ \\
& $\gamma h_{b}(1 P)$ & $8.40 \times 10^{-3}$ & $1.06 \times 10^{-4}$ \\
& $\gamma h_{b}(2 P)$ & 2.60 & $3.27 \times 10^{-2}$ \\
& $\gamma \Upsilon(1 S)$ & $5.10 \times 10^{-2}$ & $6.42 \times 10^{-4}$ \\
& $\gamma \Upsilon(2 S)$ & $9.20 \times 10^{-3}$ & $1.16 \times 10^{-4}$ \\
& $\pi \pi \eta_{b}(1 S)$ & 1.95 & $2.45 \times 10^{-2}$ \\
$\pi \pi \eta_{b}(2 S)$ & 0.34 & $4.28 \times 10^{-3}$ \\
$\operatorname{total}$ & $7.95 \mathrm{MeV}$ & 100.00 \\
\hline \hline
\end{tabular}

splitting is $\Delta m_{h f}\left[\eta_{b}(3 S)\right]=19 \mathrm{MeV}$, which follows the expected trend.

The decay widths and branching fractions of annihilation rates, radiative decays and hadronic transitions for the $\eta_{b}$ states are given in Table III. In all cases, the most important contribution to the total decay width comes from the gluon annihilation rate. Note that they are given in $\mathrm{MeV}$ whereas the rest of the quantities are given in $\mathrm{keV}$. Beyond the gluon annihilation rates, the next significant decay channel shown in Table III is the $\eta_{b}(2 S)$ into $\pi \pi \eta_{b}(1 S)$ with a width 4 times higher than the most dominant electromagnetic transitions $\eta_{b}(2 S) \rightarrow h_{b}(1 P) \gamma$ and $\eta_{b}(3 S) \rightarrow h_{b}(2 P) \gamma$. This behavior is due to the fact that we use the full expression of Eq. (B3) for the E1 radiative decays and not only the leading term. The corrections over the low energy expansion are important for these excited states. The hadronic transition of the $\eta_{b}(3 S)$ into the $\pi \pi \eta_{b}(1 S)$ final channel presents a decay width of the same order of magnitude as that of the $\eta_{b}(3 S) \rightarrow h_{b}(2 P) \gamma$ decay.

It is important to mention here that, as we will explain next, we do not expect perfect agreement with experiment for the pseudoscalar mesons. The worst situation is found for the $\eta_{b}(1 S)$ state and then it is alleviated with higher excitations. This trend is inferred from the discussion above, and the most evidence of this appears in our calculation of the total decay width of the $\eta_{b}(1 S)$ state, $20.2 \mathrm{MeV}$, which is a factor of 2 larger than the central value of the last experimental measurement [11], $\left(10.8_{-3.7-2.0}^{+4.0+4.5}\right) \mathrm{MeV}$ (see that our theoretical result lies just above the upper limit of the error bar). The reason for that is the following: Our CQM presents an OGE potential which 
has a spin-spin contact hyperfine interaction that is proportional to a Dirac delta function, conveniently regularized, at the origin (see Eqs. (A1) and (A2)). The corresponding regularization parameter was fitted to determine the hyperfine splittings between the $n^{1} S_{0}$ and $n^{3} S_{1}$ states in the different flavor sectors. While most of the physical observables are insensitive to the regularization of this delta term, those related with annihilation processes are affected because these processes are driven by short range operators $[72,73]$. The effect is very small in the ${ }^{3} S_{1}$ channel as the delta term is repulsive in this case. It is negligible for higher partial waves due to the shielding by the centrifugal barrier. However, it is sizable in the ${ }^{1} S_{0}$ channel for which the delta term is attractive and the sensitivity decreases as going up in higher excited states.

\section{B. The $h_{b}$ and $\chi_{b J}$ states}

Table II shows the predicted masses of the singlet ${ }^{1} P_{1}$ and the triplet ${ }^{3} P_{J}$ states. They are in reasonable agreement with the experimental data.

The spin-singlet $P$-wave states, $h_{b}$, are expected to lie very close in mass to the spin-weighted average of the triplet $P$-wave states, $\chi_{b J}$. This is because the hyperfine splitting in leading nonrelativistic order is proportional to the square of the wave function at the origin, which vanishes for $P$-wave states. In Table IV we compare the centroid of ${ }^{3} P_{J}$ states and the corresponding $h_{b}$ mass for the ground state and the first two excitations. One can see, on one hand, that the experimental data follow the theoretical expectations and, on the other hand, that our spin-spin interaction is negligible for $P$-wave states, as should be.

It is also important to remark that our spin-averaged centroid of the $\chi_{b J}(3 P)$ states, $10516 \mathrm{MeV}$, is compatible but slightly higher than the value collected by PDG [22]. However, the centroid is expected to be close to the $\chi_{b 1}(3 P)$ element of the spin multiplet, and the experimental measurement of the mass of this state has been recently reported by the $\mathrm{LHCb}$ [21] Collaboration with a value of 10515. $7_{-3.9-2.1}^{+2.2+1.5} \mathrm{MeV}$, which is in perfect agreement with our prediction. In addition, we calculate the intramultiplet splittings as $m_{\chi_{b 2}(3 P)}-m_{\chi_{b 1}(3 P)}=8 \mathrm{MeV}$ and $m_{\chi_{b 1}(3 P)}-m_{\chi_{b 0}(3 P)}=13 \mathrm{MeV}$.

TABLE IV. The theoretical masses, in $\mathrm{MeV}$, of the ground state and the first two excitations of $h_{b}$, compared with the spin-averaged centroid, in $\mathrm{MeV}$, of the corresponding triplet $P$-wave states. We compare with the experimental data collected in PDG [22].

\begin{tabular}{ccccc}
\hline \hline$n$ & $\begin{array}{c}m_{\text {The }}\left(h_{b}\right) \\
(\mathrm{MeV})\end{array}$ & $\begin{array}{c}m_{\operatorname{Exp}}\left(h_{b}\right)[22] \\
(\mathrm{MeV})\end{array}$ & $\begin{array}{c}\left\langle m\left(n^{3} P_{J}\right)\right\rangle_{\text {The }} \\
(\mathrm{MeV})\end{array}$ & \multicolumn{1}{c}{$\begin{array}{c}\left\langle m\left(n^{3} P_{J}\right)\right\rangle_{\text {Exp }} \\
{[22]}\end{array}$} \\
\hline 1 & 9879 & $9899.3 \pm 1.0$ & 9879 & $9899.87 \pm 0.27$ \\
2 & 10240 & $10259.8 \pm 1.2$ & 10240 & $10260.20 \pm 0.36$ \\
3 & 10516 & $\cdots$ & 10516 & $10534 \pm 9$ \\
\hline \hline
\end{tabular}

The decay widths and branching fractions of annihilation rates, radiative decays and hadronic transitions for the $h_{b}$ states are shown in Table V. We predict total decay widths of about $100 \mathrm{keV}$ for the three $h_{b}$ states. Their total decay widths are dominated by their annihilation into gluons.

The Belle Collaboration has studied very recently the processes $e^{+} e^{-} \rightarrow \Upsilon(5 S) \rightarrow h_{b}(n P) \pi^{+} \pi^{-} \rightarrow\left[\eta_{b}(m S) \gamma\right] \pi^{+} \pi^{-}$ providing branching fractions for the decays $h_{b}(1 P) \rightarrow$ $\eta_{b}(1 S) \gamma, h_{b}(2 P) \rightarrow \eta_{b}(1 S) \gamma$ and $h_{b}(2 P) \rightarrow \eta_{b}(2 S) \gamma$ [11]. One can see in Table V that our results are in reasonable agreement with experiment except for the case $h_{b}(2 P) \rightarrow$ $\eta_{b}(2 S) \gamma$ which is roughly a factor of 2 lower. It is worth mentioning that the error is bigger in this case, and the experimental figure also seems to be higher than other theoretical predictions [74].

There are four bottomonium states involved in the decay processes shown in Table $\mathrm{V}$ which are still experimentally missing. These are the $\eta_{b}(3 S), h_{b}(3 P), \eta_{b 2}(1 D)$ and $\eta_{b 2}(2 D)$. We have discussed already the $\eta_{b}(3 S)$ state, and we shall postpone for later on our discussion about the $1 D$ and $2 D$ states of the $\eta_{b 2}$ meson. The $h_{b}(3 P)$ meson has branching fractions of about $8 \%, 7 \%$ and $13 \%$ for its radiative decays into the $\eta_{b}(1 S), \eta_{b}(2 S)$ and $\eta_{b}(3 S)$ states, respectively. Since the radiative decay rates of the $\eta_{b}(3 S)$ into the already observed $h_{b}(1 P)$ and $h_{b}(2 P)$ are, respectively, 0.0084 and $2.60 \mathrm{keV}$ (see Table III), the decay chain $h_{b}(3 P) \rightarrow \eta_{b}(3 S) \gamma \rightarrow h_{b}(2 P) \gamma \gamma$ appears as the most suitable way for observing the $h_{b}(3 P)$ and $\eta_{b}(3 S)$ states. The branching fraction of the $h_{b}(3 P)$ radiative decay into $\gamma \eta_{b 2}(2 D)$ is non negligible with a value of $5 \%$ and can represent an opportunity to observe the $\eta_{b 2}(2 D)$ once the $h_{b}(3 P)$ is established.

Tables VI and VII show E1 and M1 radiative decays of the $\chi_{b J}(n P)$ states with $J=0,1,2$ and $n=1,2,3$. These Tables also show some hadronic transitions (the spinnonflip $\pi \pi$ transitions) and the annihilation rates into gluons. As one can see, the available experimental data is scarce and mostly related with the E1 radiative decays associated with the decay channels through which the $\chi_{b J}(1 P)$ and $\chi_{b J}(2 P)$ were discovered in the early eighties. Our theoretical results are in reasonable agreement with the experimental figures except for the case of the $\chi_{b 0}(2 P)$, in which our predictions of the branching fractions are much smaller than the experimental data. There could be two reasons for this. The first one could be related with an overestimation of the decay rate for the annihilation into gluons of the $\chi_{b 0}(2 P)$ state. However, This should have been reflected also in the $\chi_{b 0}(1 P)$ state and even more strongly because the smaller number of open decay channels. This is not the case as reflected in Table VI. Therefore, if we are overestimating the $\chi_{b 0}(2 P)$ annihilation into gluons, this cannot be in a dramatic way. The second possibility could be an error on the experimental measurements which have, up to now, uncertainties in the order of $50 \%$. 
TABLE V. Decay widths and branching fractions of annihilation rates, radiative decays and hadronic transitions for the $h_{b}$ states. The experimental data are from Ref. [11].

\begin{tabular}{|c|c|c|c|c|}
\hline Initial state & Final state & $\Gamma_{\text {The }}(\mathrm{keV})$ & $\mathcal{B}_{\text {The }}\left(\times 10^{-2}\right)$ & $\mathcal{B}_{\operatorname{Exp}}[11]\left(\times 10^{-2}\right)$ \\
\hline \multirow[t]{5}{*}{$h_{b}(1 P)$} & $g g g$ & 35.26 & 44.68 & $\ldots$ \\
\hline & $\gamma \eta_{b}(1 S)$ & 43.66 & 55.32 & $49.2 \pm 5.7_{-3.3}^{+5.6}$ \\
\hline & $\gamma \chi_{b 0}(1 P)$ & $8.61 \times 10^{-4}$ & $1.09 \times 10^{-3}$ & $\ldots$ \\
\hline & $\gamma \chi_{b 1}(1 P)$ & $1.15 \times 10^{-5}$ & $1.46 \times 10^{-5}$ & $\cdots$ \\
\hline & total & 78.92 & 100.00 & $\cdots$ \\
\hline \multirow[t]{9}{*}{$h_{b}(2 P)$} & $g g g$ & 52.70 & 57.82 & $\cdots$ \\
\hline & $\gamma \eta_{b}(1 S)$ & 14.90 & 16.35 & $22.3 \pm 3.8_{-3.3}^{+3.1}$ \\
\hline & $\gamma \eta_{b}(2 S)$ & 17.60 & 19.31 & $47.5 \pm 10.5_{-7.7}^{+6.8}$ \\
\hline & $\gamma \eta_{b 2}(1 D)$ & 5.36 & 5.88 & $\cdots$ \\
\hline & $\gamma \chi_{b 0}(1 P)$ & $3.64 \times 10^{-2}$ & $3.99 \times 10^{-2}$ & $\cdots$ \\
\hline & $\gamma \chi_{b 1}(1 P)$ & $1.28 \times 10^{-3}$ & $1.41 \times 10^{-3}$ & $\cdots$ \\
\hline & $\gamma \chi_{b 2}(1 P)$ & $6.91 \times 10^{-6}$ & $7.58 \times 10^{-6}$ & $\cdots$ \\
\hline & $\pi \pi h_{b}(1 P)$ & 0.54 & 0.59 & $\cdots$ \\
\hline & total & 91.14 & 100.00 & $\ldots$ \\
\hline \multirow[t]{14}{*}{$h_{b}(3 P)$} & $g g g$ & 62.16 & 64.91 & $\ldots$ \\
\hline & $\gamma \eta_{b}(1 S)$ & 7.96 & 8.31 & $\cdots$ \\
\hline & $\gamma \eta_{b}(2 S)$ & 6.86 & 7.16 & $\cdots$ \\
\hline & $\gamma \eta_{b}(3 S)$ & 12.27 & 12.81 & $\cdots$ \\
\hline & $\gamma \eta_{b 2}(1 D)$ & 0.35 & 0.37 & $\cdots$ \\
\hline & $\gamma \eta_{b 2}(2 D)$ & 4.72 & 4.93 & $\ldots$ \\
\hline & $\gamma \chi_{b 0}(1 P)$ & $3.77 \times 10^{-3}$ & $3.94 \times 10^{-3}$ & $\cdots$ \\
\hline & $\gamma \chi_{b 1}(1 P)$ & $1.23 \times 10^{-3}$ & $1.28 \times 10^{-3}$ & $\cdots$ \\
\hline & $\gamma \chi_{b 2}(1 P)$ & $5.10 \times 10^{-5}$ & $5.33 \times 10^{-5}$ & $\cdots$ \\
\hline & $\gamma \chi_{b 0}(2 P)$ & $1.71 \times 10^{-3}$ & $1.79 \times 10^{-3}$ & $\cdots$ \\
\hline & $\gamma \chi_{b 1}(2 P)$ & $5.97 \times 10^{-4}$ & $6.23 \times 10^{-4}$ & $\cdots$ \\
\hline & $\gamma \chi_{b 2}(2 P)$ & $7.37 \times 10^{-6}$ & $7.70 \times 10^{-6}$ & $\cdots$ \\
\hline & $\pi \pi h_{b}(1 P)$ & 1.44 & 1.50 & $\ldots$ \\
\hline & total & 95.76 & 100.00 & $\ldots$ \\
\hline
\end{tabular}

One can inferred from the Tables VI and VII that the $\chi_{b 1}(1 P, 2 P, 3 P)$ and $\chi_{b 2}(1 P, 2 P, 3 P)$ mesons have total decay widths of around $100-150 \mathrm{keV}$ whereas the $\chi_{b 0}(1 P, 2 P, 3 P)$ states have total decay widths of about $2-2.5 \mathrm{MeV}$. The contribution of the decay rate into gluons is $99 \%$ in the case of the $\chi_{b 0}$ states compared with the 70$80 \%$ for the $\chi_{b 1}$ and $\chi_{b 2}$ mesons.

Special attention deserves Table VII in which we have collected the decay properties of the spin-triplet $3 P$-wave states. We hope that the theoretical data shown in Table VII help experimentalists in carrying out an intensive study of them. Some of their radiative decays are dominant, with rates in the order of few $\mathrm{keV}$. Therefore, these transitions still seem to be the best way for disentangling the fine mass splittings. The spin-nonflip $\pi \pi$ transitions $\chi_{b 0}(3 P) \rightarrow$ $\chi_{b 0}(1 P), \chi_{b 1}(3 P) \rightarrow \chi_{b 1}(1 P)$ and $\chi_{b 2}(3 P) \rightarrow \chi_{b 2}(1 P)$ have decay rates lower but in the same order of magnitude, around $1-2 \mathrm{keV}$, and thus can be also used for studying the $3 P$ spin-triplet states.

We finish this Section calling the attention of the reader to the fact that our prediction of the spin-nonflip $\pi \pi$ transitions between $\chi_{b J}$ states follow a particular path: the decay rates between states with $J_{i}=J_{f}$ are orders of magnitude higher than those with $J_{i} \neq J_{f}$. This is so because the first type of transitions goes through the term with the coefficient $C_{1}$ of Eq. (B29) whereas the second type of transitions involve only the term of Eq. (B29) with constant $C_{2}$ that is much smaller. The constant $C_{1}$ is fitted through the $\Upsilon(2 S) \rightarrow \pi \pi \Upsilon(1 S)$ decay whereas the coefficient $C_{2}$ is fitted through the transition $\Upsilon_{2}(1 D) \rightarrow$ $\pi \pi \Upsilon(1 S)$. It is remarkable that, as seen in Table VI, we obtain good agreement in those cases in which experimental data are available.

\section{The $S$-wave $\Upsilon$ levels}

The success of QCD-inspired potentials is due largely to the fruitful description and prediction of the properties of the $S$-wave $\psi$ and $\Upsilon$ states. One can see in Table II that the masses of $\Upsilon(1 S), \Upsilon(2 S)$ and $\Upsilon(3 S)$ located experimentally at $9.46,10.02$ and $10.35 \mathrm{GeV}$ are reasonably well reproduce in our quark model: 9.50, 10.02 and $10.35 \mathrm{GeV}$, respectively.

We show in Table VIII the decay widths and branching fractions of annihilation rates, radiative decays and hadronic transitions for the $\Upsilon(1 S), \Upsilon(2 S)$ and $\Upsilon(3 S)$ states. In 
TABLE VI. Decay widths and branching fractions of annihilation rates, radiative decays and hadronic transitions for the $\chi_{b J}$ states. The experimental data are from Ref. [22].

\begin{tabular}{|c|c|c|c|c|}
\hline Initial state & Final state & $\Gamma_{\text {The }}(\mathrm{keV})$ & $\mathcal{B}_{\text {The }}\left(\times 10^{-2}\right)$ & $\mathcal{B}_{\text {Exp }}[22]\left(\times 10^{-2}\right)$ \\
\hline \multirow{4}{*}{$\overline{\chi_{b 0}(1 P)}$} & $g g$ & $2.00 \mathrm{MeV}$ & 98.61 & $\ldots$ \\
\hline & $\gamma \gamma$ & 0.12 & $5.91 \times 10^{-3}$ & $\ldots$ \\
\hline & $\gamma \Upsilon(1 S)$ & 28.07 & 1.38 & $1.76 \pm 0.30 \pm 0.18$ \\
\hline & total & $2.03 \mathrm{MeV}$ & 100.00 & $\ldots$ \\
\hline \multirow[t]{3}{*}{$\chi_{b 1}(1 P)$} & $q \bar{q}+g$ & 71.53 & 66.73 & $\cdots$ \\
\hline & $\gamma \Upsilon(1 S)$ & 35.66 & 33.27 & $33.9 \pm 2.2$ \\
\hline & total & 107.19 & 100.00 & $\ldots$ \\
\hline \multirow[t]{5}{*}{$\chi_{b 2}(1 P)$} & $g g$ & 83.69 & 68.13 & $\cdots$ \\
\hline & $\gamma \gamma$ & $3.08 \times 10^{-3}$ & $2.51 \times 10^{-3}$ & $\cdots$ \\
\hline & $\gamma \Upsilon \mathbf{\Upsilon}(1 S)$ & 39.15 & 31.87 & $19.1 \pm 1.2$ \\
\hline & $\gamma h_{b}(1 P)$ & $8.88 \times 10^{-5}$ & $7.23 \times 10^{-5}$ & $\ldots$ \\
\hline & total & 122.84 & 100.00 & $\cdots$ \\
\hline \multirow[t]{9}{*}{$\chi_{b 0}(2 P)$} & $g g$ & $2.37 \mathrm{MeV}$ & 99.17 & $\ldots$ \\
\hline & $\gamma \gamma$ & 0.14 & $5.85 \times 10^{-3}$ & $\cdots$ \\
\hline & $\gamma \Upsilon(1 S)$ & 5.44 & 0.23 & $0.9 \pm 0.6$ \\
\hline & $\gamma \Upsilon(2 S)$ & 12.80 & 0.54 & $4.6 \pm 2.1$ \\
\hline & $\gamma \Upsilon(1 D)$ & 0.74 & $3.09 \times 10^{-2}$ & $\ldots$ \\
\hline & $\gamma h_{b}(1 P)$ & $2.39 \times 10^{-3}$ & $9.99 \times 10^{-5}$ & $\cdots$ \\
\hline & $\pi \pi \chi_{b 0}(1 P)$ & 0.72 & $3.01 \times 10^{-2}$ & $\cdots$ \\
\hline & $\pi \pi \chi_{b 2}(1 P)$ & $4.08 \times 10^{-5}$ & $1.71 \times 10^{-6}$ & $\cdots$ \\
\hline & total & $2.39 \mathrm{MeV}$ & 100.00 & $\cdots$ \\
\hline \multirow[t]{9}{*}{$\chi_{b 1}(2 P)$} & $q \bar{q}+g$ & 106.14 & 79.57 & $\ldots$ \\
\hline & $\gamma \Upsilon(1 S)$ & 9.13 & 6.84 & $9.2 \pm 0.8$ \\
\hline & $\gamma \Upsilon(2 S)$ & 15.89 & 11.91 & $19.9 \pm 1.9$ \\
\hline & $\gamma \Upsilon(1 D)$ & 0.41 & 0.31 & $\ldots$ \\
\hline & $\gamma \Upsilon_{2}(1 D)$ & 1.26 & 0.95 & $\cdots$ \\
\hline & $\gamma h_{b}(1 P)$ & $1.67 \times 10^{-4}$ & $1.25 \times 10^{-4}$ & $\ldots$ \\
\hline & $\pi \pi \chi_{b 1}(1 P)$ & 0.57 & 0.43 & $0.91 \pm 0.13$ \\
\hline & $\pi \pi \chi_{b 2}(1 P)$ & $1.94 \times 10^{-4}$ & $1.45 \times 10^{-4}$ & $\ldots$ \\
\hline & total & 133.40 & 100.00 & $\cdots$ \\
\hline \multirow{13}{*}{$\chi_{b 2}(2 P)$} & $g g$ & 104.26 & 76.62 & $\ldots$ \\
\hline & $\gamma \gamma$ & $3.84 \times 10^{-3}$ & $2.82 \times 10^{-3}$ & $\cdots$ \\
\hline & $\gamma \Upsilon(1 S)$ & 11.38 & 8.36 & $7.0 \pm 0.7$ \\
\hline & $\gamma \Upsilon(2 S)$ & 17.50 & 12.86 & $10.6 \pm 2.6$ \\
\hline & $\gamma \Upsilon(1 D)$ & $2.09 \times 10^{-2}$ & $1.54 \times 10^{-2}$ & $\cdots$ \\
\hline & $\gamma \Upsilon_{2}(1 D)$ & 0.35 & 0.26 & $\cdots$ \\
\hline & $\gamma \Upsilon_{3}(1 D)$ & 2.06 & 1.51 & $\cdots$ \\
\hline & $\gamma h_{b}(1 P)$ & $1.78 \times 10^{-3}$ & $1.31 \times 10^{-3}$ & $\cdots$ \\
\hline & $\gamma h_{b}(2 P)$ & $2.86 \times 10^{-5}$ & $2.10 \times 10^{-5}$ & $\cdots$ \\
\hline & $\pi \pi \chi_{b 0}(1 P)$ & $8.49 \times 10^{-6}$ & $6.24 \times 10^{-6}$ & $\cdots$ \\
\hline & $\pi \pi \chi_{b 1}(1 P)$ & $6.06 \times 10^{-4}$ & $4.45 \times 10^{-4}$ & $\ldots$ \\
\hline & $\pi \pi \chi_{b 2}(1 P)$ & 0.49 & 0.36 & $0.51 \pm 0.09$ \\
\hline & total & 136.07 & 100.00 & $\ldots$ \\
\hline
\end{tabular}

order to avoid unnecessary uncertainties in the calculated branching fractions, we use in these cases the total decay widths available in PDG [22].

The annihilation rates of the $\Upsilon(1 S)$ state are slightly lower than the experimental data but in reasonable agreement. Unfortunately, there is no experimental data associated with the only radiative decay of the $\Upsilon(1 S)$ state. The $\Upsilon(1 S) \rightarrow \gamma \eta_{b}(1 S)$ is an M1 transition and thus it is suppressed with respect the E1 decays and much more difficult to measure. This can be observed in the cases of the $\Upsilon(2 S)$ and $\Upsilon(3 S)$ where transitions into $\eta_{b}$ states are orders of magnitude smaller than the transitions into $\chi_{b J}$ states. Using pNRQCD [75], the authors of Ref. [76] have recently computed the decay rate of the $\Upsilon(1 S) \rightarrow \gamma \eta_{b}(1 S)$ transition reporting a value of $(0.01518 \pm 0.00051) \mathrm{keV}$, which is higher than our quark model prediction of $\sim 0.0093 \mathrm{keV}$. This decay rate is extremely sensitive to the masses of the $\Upsilon(1 S)$ and $\eta_{b}(1 S)$ mesons. If we compute the decay width 
TABLE VII. (Continuation) Decay widths and branching fractions of annihilation rates, radiative decays and hadronic transitions for the $\chi_{b J}$ states. There is no experimental data available.

\begin{tabular}{|c|c|c|c|}
\hline Initial state & Final state & $\Gamma_{\text {The }}(\mathrm{keV})$ & $\mathcal{B}_{\text {The }}\left(\times 10^{-2}\right.$ \\
\hline \multirow[t]{10}{*}{$\chi_{b 0}(3 P)$} & $g g$ & $2.46 \mathrm{MeV}$ & 99.26 \\
\hline & $\gamma \gamma$ & 0.15 & $6.06 \times 10^{-3}$ \\
\hline & $\gamma \Upsilon(1 S)$ & 1.99 & $8.04 \times 10^{-2}$ \\
\hline & $\gamma \Upsilon(2 S)$ & 2.99 & 0.12 \\
\hline & $\gamma \Upsilon(3 S)$ & 8.50 & 0.34 \\
\hline & $\gamma \Upsilon(1 D)$ & $3.59 \times 10^{-2}$ & $1.45 \times 10^{-3}$ \\
\hline & $\gamma \Upsilon(2 D)$ & 3.50 & 0.14 \\
\hline & $\pi \pi \chi_{b 0}(1 P)$ & 1.16 & $4.69 \times 10^{-2}$ \\
\hline & $\pi \pi \chi_{b 2}(1 P)$ & $4.28 \times 10^{-3}$ & $1.73 \times 10^{-4}$ \\
\hline & total & $2.47 \mathrm{MeV}$ & 100.00 \\
\hline \multirow[t]{11}{*}{$\chi_{b 1}(3 P)$} & $q \bar{q}+g$ & 124.53 & 83.59 \\
\hline & $\gamma \Upsilon(1 S)$ & 4.17 & 2.80 \\
\hline & $\gamma \Upsilon(2 S)$ & 4.58 & 3.07 \\
\hline & $\gamma \Upsilon(3 S)$ & 9.62 & 6.46 \\
\hline & $\gamma \Upsilon(1 D)$ & $4.80 \times 10^{-2}$ & $3.22 \times 10^{-2}$ \\
\hline & $\gamma \Upsilon(2 D)$ & 1.26 & 0.85 \\
\hline & $\gamma \Upsilon_{2}(1 D)$ & 0.11 & $7.38 \times 10^{-2}$ \\
\hline & $\gamma \Upsilon_{2}(2 D)$ & 3.34 & 2.24 \\
\hline & $\pi \pi \chi_{b 1}(1 P)$ & 1.32 & 0.89 \\
\hline & $\pi \pi \chi_{b 2}(1 P)$ & $4.55 \times 10^{-3}$ & $3.05 \times 10^{-3}$ \\
\hline & total & 148.98 & 100.00 \\
\hline \multirow[t]{15}{*}{$\chi_{b 2}(3 P)$} & $g g$ & 111.45 & 79.56 \\
\hline & $\gamma \gamma$ & $4.10 \times 10^{-3}$ & $2.93 \times 10^{-3}$ \\
\hline & $\gamma \Upsilon(1 S)$ & 5.65 & 4.03 \\
\hline & $\gamma \Upsilon(2 S)$ & 5.62 & 4.01 \\
\hline & $\gamma \Upsilon(3 S)$ & 10.38 & 7.41 \\
\hline & $\gamma \Upsilon(1 D)$ & $3.38 \times 10^{-3}$ & $2.41 \times 10^{-3}$ \\
\hline & $\gamma \Upsilon(2 D)$ & 0.18 & 0.13 \\
\hline & $\gamma \Upsilon_{2}(1 D)$ & $4.41 \times 10^{-2}$ & $3.15 \times 10^{-2}$ \\
\hline & $\gamma \Upsilon_{2}(2 D)$ & 0.79 & 0.56 \\
\hline & $\gamma \Upsilon_{3}(1 D)$ & 0.21 & 0.15 \\
\hline & $\gamma \Upsilon_{3}(2 D)$ & 4.16 & 2.97 \\
\hline & $\pi \pi \chi_{b 0}(1 P)$ & $1.88 \times 10^{-3}$ & $1.34 \times 10^{-3}$ \\
\hline & $\pi \pi \chi_{b 1}(1 P)$ & $4.37 \times 10^{-3}$ & $3.12 \times 10^{-3}$ \\
\hline & $\pi \pi \chi_{b 2}(1 P)$ & 1.59 & 1.14 \\
\hline & total & 140.09 & 100.00 \\
\hline
\end{tabular}

using the masses predicted by pNRQCD, our result is $0.014 \mathrm{keV}$ and agrees with the pNRQCD value.

As one can see in Table VIII, all branching fractions of the $\Upsilon(2 S)$ state which are related with annihilation, E1 and M1 radiative decays and even the spin-nonflip $\pi \pi$ transitions are in reasonable agreement with the experimental data. In Ref. [12], Lees et al. have performed a study of radiative transitions between bottomonium states with a huge amount of events recorded by the BABAR detector at the PEP-II $B$ factory at SLAC. Among their measurements, a value of the $\Upsilon(2 S) \rightarrow \gamma \eta_{b}(1 S)$ decay rate is reported and agrees, within errors, with our theoretical figure (see Table VIII). However, it is important to emphasize that this decay rate is far from being to be well established. On the experimental side, the PDG of 2014 collects the branching fraction $(3.9 \pm 1.5) \times 10^{-4}$ from an early measurement of the BABAR Collaboration [77] despite having the measurement of 2011 reported in Ref. [12]. On the theoretical side, there are values in lattice NRQCD [78], $(5.4 \pm 1.8) \times 10^{-4}$, in continuum pNRQCD [76], $(1.88 \pm 8.34) \times 10^{-4}$, and within quark models ranging from $0.05 \times 10^{-4}$ to $18 \times 10^{-4}$.

The nice agreement with experimental data seems to change for the $\Upsilon(3 S)$ state. While we reproduce the branching fractions for the radiative decays of the $\Upsilon(3 S)$ into $\chi_{b J}(2 P)$ states and also for the 2-pion decays into $\Upsilon(1 S)$ and $\Upsilon(2 S)$ states, we are only able to give the correct order of magnitude for the E1 radiative decays of the $\Upsilon(3 S)$ into $\chi_{b J}(1 P)$ states, and the prediction for the annihilation rate into gluons seems to be much larger than the experimental figure.

In December of 2014, the BABAR Collaboration published an experimental work [79] in which 121 million of $\Upsilon(3 S)$ and 98 million of $\Upsilon(2 S)$ mesons were used to perform a study of radiative transitions involving the $\chi_{b J}(1 P, 2 P)$ states. This work includes the best observational significance of some transitions and provides the most up-to-date derived branching fractions in the bottomonium system. Table IX summarizes their primary results and compares them with our theoretical values. Our results are compatible within experimental errors in most cases but some discrepancies are also found.

Above the $B \bar{B}$ threshold, there are three more states well established in the PDG with quantum number $J^{P C}=1^{--}$. They are the so-called $\Upsilon(4 S), \Upsilon(10860)$ and $\Upsilon(11020)$, being the last two natural candidates for the $\Upsilon(5 S)$ and $\Upsilon(6 S)$, respectively. The $B$ factories scanned again the energy range above the open-bottom threshold. The $B A B A R$ Collaboration [80] performed a comprehensive scan between 10.54 and $11.2 \mathrm{GeV}$, followed by an eightpoint scan in the proximity of the $\Upsilon(6 S)$ peak. The Belle Collaboration [81] acquired nine points over 10.80$11.02 \mathrm{GeV}$, as well as spread over seven additional points more focused on the $\Upsilon(5 S)$ peak. Both scans suggest that the simple Breit-Wigner parametrization, previously used to model the peaks observed in the CLEO [7] and CUSB [8] scans, is not good enough for the description of the complex dynamics in the proximity of the $B^{(*)} \bar{B}^{(*)}$ and $B_{s}^{(*)} \bar{B}_{s}{ }^{(*)}$ thresholds. The new data points on $R_{b}=$ $\sigma(b \bar{b}) / \sigma(\mu \mu)$ are better modeled assuming a flat $b \bar{b}$ continuum contribution which interferes constructively with the $\Upsilon(5 S)$ and $\Upsilon(6 S)$ Breit-Wigner resonances, and a second flat contribution which adds incoherently. Such fits alter the PDG results on the $\Upsilon(5 S)$ and $\Upsilon(6 S)$ peaks. Table $\mathrm{X}$ compares the theoretical prediction with the new parameters reported by $B A B A R$ and Belle, but also with the PDG's values.

Table XI shows the decay widths and branching fractions of annihilation rates, radiative decays and hadronic transitions for the $\Upsilon(4 S), \Upsilon(10860)$ and $\Upsilon(11020)$ states. The 
TABLE VIII. Decay widths and branching fractions of annihilation rates, radiative decays and hadronic transitions for the $\Upsilon(1 S), \Upsilon(2 S)$ and $\Upsilon(3 S)$ states. The experimental data are from Refs. [12,22]. In this case, we have calculated the branching fractions using the experimental total decay widths of PDG2014.

\begin{tabular}{|c|c|c|c|c|}
\hline Initial state & Final state & $\Gamma_{\text {The }}(\mathrm{keV})$ & $\mathcal{B}_{\text {The }}\left(\times 10^{-2}\right)$ & $\mathcal{B}_{\operatorname{Exp}}\left(\times 10^{-2}\right)$ \\
\hline \multirow[t]{5}{*}{$\overline{\Upsilon(1 S)}$} & $e^{+} e^{-}$ & 0.71 & 1.31 & $2.38 \pm 0.11$ \\
\hline & $3 g$ & 41.63 & 77.06 & $81.7 \pm 0.7$ \\
\hline & $\gamma g g$ & 0.79 & 1.46 & $2.2 \pm 0.6$ \\
\hline & $3 \gamma$ & $3.44 \times 10^{-6}$ & $6.37 \times 10^{-6}$ & $\ldots$ \\
\hline & $\gamma \eta_{b}(1 S)$ & $9.34 \times 10^{-3}$ & $1.73 \times 10^{-2}$ & $\ldots$ \\
\hline \multirow[t]{10}{*}{$\Upsilon(2 S)$} & $e^{+} e^{-}$ & 0.37 & 1.16 & $1.91 \pm 0.16$ \\
\hline & $3 g$ & 24.25 & 75.83 & $58.8 \pm 1.2$ \\
\hline & $\gamma g g$ & 0.46 & 1.44 & $8.8 \pm 1.1$ \\
\hline & $3 \gamma$ & $2.00 \times 10^{-6}$ & $6.25 \times 10^{-6}$ & $\ldots$ \\
\hline & $\gamma \chi_{b 0}(1 P)$ & 1.09 & 3.41 & $3.8 \pm 0.4$ \\
\hline & $\gamma \chi_{b 1}(1 P)$ & 1.84 & 5.75 & $6.9 \pm 0.4$ \\
\hline & $\gamma \chi_{b 2}(1 P)$ & 2.08 & 6.50 & $7.15 \pm 0.35$ \\
\hline & $\gamma \eta_{b}(1 S)$ & $5.65 \times 10^{-2}$ & 0.18 & $0.11 \pm 0.04_{-0.05}^{+0.07}[12]$ \\
\hline & $\gamma \eta_{b}(2 S)$ & $5.80 \times 10^{-4}$ & $1.81 \times 10^{-3}$ & $\ldots$ \\
\hline & $\pi \pi \Upsilon(1 S)$ & 8.57 & 26.80 & $26.45 \pm 0.48$ \\
\hline \multirow[t]{15}{*}{$\Upsilon(3 S)$} & $e^{+} e^{-}$ & 0.27 & 1.33 & $2.18 \pm 0.20$ \\
\hline & $3 g$ & 18.76 & 92.32 & $35.7 \pm 2.6$ \\
\hline & $\gamma g g$ & 0.36 & 1.77 & $0.97 \pm 0.18$ \\
\hline & $3 \gamma$ & $1.55 \times 10^{-6}$ & $7.63 \times 10^{-6}$ & $\ldots$ \\
\hline & $\gamma \chi_{b 0}(1 P)$ & 0.15 & 0.74 & $0.27 \pm 0.04$ \\
\hline & $\gamma \chi_{b 1}(1 P)$ & 0.16 & 0.79 & $0.09 \pm 0.05$ \\
\hline & $\gamma \chi_{b 2}(1 P)$ & $8.27 \times 10^{-2}$ & 0.41 & $0.99 \pm 0.13$ \\
\hline & $\gamma \chi_{b 0}(2 P)$ & 1.21 & 5.96 & $5.9 \pm 0.6$ \\
\hline & $\gamma \chi_{b 1}(2 P)$ & 2.13 & 10.48 & $12.6 \pm 1.2$ \\
\hline & $\gamma \chi_{b 2}(2 P)$ & 2.56 & 12.60 & $13.1 \pm 1.6$ \\
\hline & $\gamma \eta_{b}(1 S)$ & $5.70 \times 10^{-2}$ & 0.28 & $0.058 \pm 0.016_{-0.016}^{+0.014}$ \\
\hline & $\gamma \eta_{b}(2 S)$ & $1.10 \times 10^{-2}$ & $5.41 \times 10^{-2}$ & $<0.062$ \\
\hline & $\gamma \eta_{b}(3 S)$ & $6.58 \times 10^{-4}$ & $3.24 \times 10^{-3}$ & $\cdots$ \\
\hline & $\pi \pi \Upsilon(1 S)$ & 1.77 & 8.71 & $6.57 \pm 0.15$ \\
\hline & $\pi \pi \Upsilon(2 S)$ & 0.42 & 2.07 & $4.67 \pm 0.23$ \\
\hline
\end{tabular}

TABLE IX. Radiative decay chains of the $\Upsilon(2 S)$ and $\Upsilon(3 S)$ states involving the $\chi_{b J}(1 P, 2 P)$ mesons. The branching fractions are $\mathcal{B}_{1}=\mathcal{B}\left(n^{3} S_{1} \rightarrow m^{3} P_{J}+\gamma\right), \mathcal{B}_{2}=\mathcal{B}\left(m^{3} P_{J} \rightarrow n^{\prime 3} S_{1}+\gamma\right)$, and $\mathcal{B}_{3}=\mathcal{B}\left(n^{\prime 3} S_{1} \rightarrow \mu^{+} \mu^{-}\right)$. For the theoretical calculation, we take the branching fraction $\mathcal{B}_{3}$ from PDG2014. The experimental data is taken from Ref. [79].

\begin{tabular}{|c|c|c|c|c|c|}
\hline Decay chain & $\mathcal{B}_{1}(\%)$ & $\mathcal{B}_{2}(\%)$ & $\mathcal{B}_{3}(\%)$ & $\mathcal{B}_{\text {The }}\left(10^{-4}\right)$ & $\mathcal{B}_{\operatorname{Exp}}[79]\left(10^{-4}\right)$ \\
\hline $2^{3} S_{1} \rightarrow 1^{3} P_{0} \rightarrow 1^{3} S_{1}$ & 3.41 & 1.38 & 2.48 & 0.12 & $0.29_{-0.14-0.08}^{+0.17+0.01}$ \\
\hline $2^{3} S_{1} \rightarrow 1^{3} P_{1} \rightarrow 1^{3} S_{1}$ & 5.75 & 33.27 & 2.48 & 4.74 & $6.86_{-0.45-0.35}^{+0.47+0.44}$ \\
\hline $2^{3} S_{1} \rightarrow 1^{3} P_{2} \rightarrow 1^{3} S_{1}$ & 6.50 & 31.87 & 2.48 & 5.14 & $3.63_{-0.34-0.19}^{+0.36+0.18}$ \\
\hline $3^{3} S_{1} \rightarrow 2^{3} P_{0} \rightarrow 2^{3} S_{1}$ & 5.96 & 0.54 & 1.93 & 0.062 & $0.66_{-0.40-0.03}^{+0.49+0.20}$ \\
\hline $3^{3} S_{1} \rightarrow 2^{3} P_{1} \rightarrow 2^{3} S_{1}$ & 10.48 & 11.91 & 1.93 & 2.41 & $4.95_{-0.70-0.24}^{+0.75+1.01}$ \\
\hline $3^{3} S_{1} \rightarrow 2^{3} P_{2} \rightarrow 2^{3} S_{1}$ & 12.60 & 12.86 & 1.93 & 3.13 & $3.22_{-0.53-0.71}^{+0.58+0.16}$ \\
\hline $3^{3} S_{1} \rightarrow 2^{3} P_{0} \rightarrow 1^{3} S_{1}$ & 5.96 & 0.23 & 2.48 & 0.034 & $0.17_{-0.14-0.12}^{+0.15+0.01}$ \\
\hline $3^{3} S_{1} \rightarrow 2^{3} P_{1} \rightarrow 1^{3} S_{1}$ & 10.48 & 6.84 & 2.48 & 1.78 & $3.52_{-0.27-0.18}^{+0.28+0.17}$ \\
\hline $3^{3} S_{1} \rightarrow 2^{3} P_{2} \rightarrow 1^{3} S_{1}$ & 12.60 & 8.36 & 2.48 & 2.61 & $1.95_{-0.21-0.16}^{+0.22+0.10}$ \\
\hline $3^{3} S_{1} \rightarrow 1^{3} P_{0} \rightarrow 1^{3} S_{1}$ & 0.74 & 1.38 & 2.48 & 0.025 & $\cdots$ \\
\hline $3^{3} S_{1} \rightarrow 1^{3} P_{1} \rightarrow 1^{3} S_{1}$ & 0.79 & 33.27 & 2.48 & 0.65 & $1.16_{-0.67-0.16}^{+0.78+0.14}$ \\
\hline $3^{3} S_{1} \rightarrow 1^{3} P_{2} \rightarrow 1^{3} S_{1}$ & 0.41 & 31.87 & 2.48 & 0.32 & $4.68_{-0.92}^{+0.99} \pm 0.37$ \\
\hline
\end{tabular}


TABLE X. New masses, in $\mathrm{MeV}$, reported by the BABAR and Belle Collaborations for the $\Upsilon(5 S)$ and $\Upsilon(6 S)$ states. We compare with our theoretical results and the current PDG2014 values.

\begin{tabular}{lcccc}
\hline \hline$\Upsilon(n S)$ & Theory & BABAR [80] & Belle [81] & PDG2014 [22] \\
\hline $5 S$ & 10818 & $10876 \pm 2$ & $10879 \pm 3$ & $10876 \pm 11$ \\
$6 S$ & 10995 & $10996 \pm 2$ & $\cdots$ & $11019 \pm 8$ \\
\hline \hline
\end{tabular}

experimental data are from Refs. [12,22]. We have again used the experimental total decay widths reported by PDG [22] in order to calculate the theoretical branching fractions. The theoretical total decay widths can be found on
Table XII which agrees well with the experimental values for the $\Upsilon(4 S)$ and $\Upsilon(11020)$ states. Since the experimental data is scarce, we can only comment interesting features of our theoretical results. The $\Upsilon(n S)$ with $n=4,5,6$ have radiative decays into the $\chi_{b J}(1 P, 2 P, 3 P)$ states whose widths go from 0.01 to $1.5 \mathrm{keV}$. Their M1 radiative decays into the $\eta_{b}$ states are 2-3 orders of magnitude smaller.

We compare in Table XI the spin-nonflip $\pi \pi$ hadronic transitions of the $\Upsilon(4 S), \Upsilon(10860)$ and $\Upsilon(11020)$ states for which experimental data are available. One can see that our values agree reasonably well with the experimental ones except in the case of the $\Upsilon(10860)$. We have seen in Refs. $[82,83]$ that the presence of hybrid mesons close in

TABLE XI. Decay widths and branching fractions of annihilation rates, radiative decays and hadronic transitions for the $\Upsilon(4 S), \Upsilon(10860)$ and $\Upsilon(11020)$ states. The experimental data are from Refs. [12,22]. In this case, we have calculated the branching fractions using the experimental total decay widths of PDG2014.

\begin{tabular}{|c|c|c|c|c|}
\hline Initial state & Final state & $\Gamma_{\text {The }}(\mathrm{keV})$ & $\mathcal{B}_{\text {The }}\left(\times 10^{-2}\right)$ & $\mathcal{B}_{\operatorname{Exp}}\left(\times 10^{-2}\right)$ \\
\hline \multirow[t]{18}{*}{$\mathbf{\Upsilon}(4 S)$} & $e^{+} e^{-}$ & 0.21 & $1.02 \times 10^{-3}$ & $(1.57 \pm 0.08) \times 10^{-3}$ \\
\hline & $3 g$ & 15.58 & $7.60 \times 10^{-2}$ & $\ldots$ \\
\hline & $\gamma g g$ & 0.30 & $1.46 \times 10^{-3}$ & $\ldots$ \\
\hline & $3 \gamma$ & $1.29 \times 10^{-6}$ & $6.29 \times 10^{-9}$ & $\ldots$ \\
\hline & $\gamma \chi_{b 0}(1 P)$ & $5.88 \times 10^{-2}$ & $2.87 \times 10^{-4}$ & $\ldots$ \\
\hline & $\gamma \chi_{b 1}(1 P)$ & $4.74 \times 10^{-2}$ & $2.31 \times 10^{-4}$ & $\cdots$ \\
\hline & $\gamma \chi_{b 2}(1 P)$ & $1.20 \times 10^{-2}$ & $5.85 \times 10^{-5}$ & $\ldots$ \\
\hline & $\gamma \chi_{b 0}(2 P)$ & 0.17 & $8.29 \times 10^{-4}$ & $\ldots$ \\
\hline & $\gamma \chi_{b 1}(2 P)$ & 0.18 & $8.78 \times 10^{-4}$ & $\ldots$ \\
\hline & $\gamma \chi_{b 2}(2 P)$ & 0.11 & $5.37 \times 10^{-4}$ & $\cdots$ \\
\hline & $\gamma \chi_{b 0}(3 P)$ & 0.61 & $2.98 \times 10^{-3}$ & $\ldots$ \\
\hline & $\gamma \chi_{b 1}(3 P)$ & 1.17 & $5.71 \times 10^{-3}$ & $\cdots$ \\
\hline & $\gamma \chi_{b 2}(3 P)$ & 1.45 & $7.07 \times 10^{-3}$ & $\ldots$ \\
\hline & $\gamma \eta_{b}(1 S)$ & $4.98 \times 10^{-2}$ & $2.43 \times 10^{-4}$ & $\ldots$ \\
\hline & $\gamma \eta_{b}(2 S)$ & $1.24 \times 10^{-2}$ & $6.05 \times 10^{-5}$ & $\cdots$ \\
\hline & $\gamma \eta_{b}(3 S)$ & $3.88 \times 10^{-3}$ & $1.89 \times 10^{-5}$ & $\ldots$ \\
\hline & $\pi \pi \Upsilon(1 S)$ & 6.02 & $2.94 \times 10^{-2}$ & $(1.22 \pm 0.09) \times 10^{-2}$ \\
\hline & $\pi \pi \Upsilon(2 S)$ & 0.24 & $1.17 \times 10^{-3}$ & $(1.29 \pm 0.20) \times 10^{-2}$ \\
\hline \multirow[t]{17}{*}{$\Upsilon(10860)$} & $e^{+} e^{-}$ & 0.18 & $3.27 \times 10^{-4}$ & $(5.6 \pm 3.1) \times 10^{-4}$ \\
\hline & $3 g$ & 13.33 & $2.42 \times 10^{-2}$ & $\ldots$ \\
\hline & $\gamma g g$ & 0.25 & $4.55 \times 10^{-4}$ & $\ldots$ \\
\hline & $3 \gamma$ & $1.10 \times 10^{-6}$ & $2.00 \times 10^{-9}$ & $\cdots-$ \\
\hline & $\gamma \chi_{b 0}(1 P)$ & $6.85 \times 10^{-2}$ & $1.25 \times 10^{-4}$ & $\ldots$ \\
\hline & $\gamma \chi_{b 1}(1 P)$ & $6.29 \times 10^{-2}$ & $1.14 \times 10^{-4}$ & $\ldots$ \\
\hline & $\gamma \chi_{b 2}(1 P)$ & $2.26 \times 10^{-2}$ & $4.11 \times 10^{-5}$ & $\cdots$ \\
\hline & $\gamma \chi_{b 0}(2 P)$ & 0.22 & $4.00 \times 10^{-4}$ & $\ldots$ \\
\hline & $\gamma \chi_{b 1}(2 P)$ & 0.26 & $4.73 \times 10^{-4}$ & $\cdots$ \\
\hline & $\gamma \chi_{b 2}(2 P)$ & 0.18 & $3.27 \times 10^{-4}$ & $\ldots$ \\
\hline & $\gamma \chi_{b 0}(3 P)$ & 0.80 & $1.45 \times 10^{-3}$ & $\ldots$ \\
\hline & $\gamma \chi_{b 1}(3 P)$ & 1.35 & $2.45 \times 10^{-3}$ & $\ldots$ \\
\hline & $\gamma \chi_{b 2}(3 P)$ & 1.42 & $2.58 \times 10^{-3}$ & $\ldots$ \\
\hline & $\gamma \eta_{b}(1 S)$ & $5.97 \times 10^{-2}$ & $1.09 \times 10^{-4}$ & $\ldots$ \\
\hline & $\gamma \eta_{b}(2 S)$ & $2.17 \times 10^{-2}$ & $3.95 \times 10^{-5}$ & $\ldots$ \\
\hline & $\gamma \eta_{b}(3 S)$ & $1.37 \times 10^{-2}$ & $2.49 \times 10^{-5}$ & $\ldots$ \\
\hline & $\pi \pi \Upsilon(1 S)$ & 12.82 & $2.33 \times 10^{-2}$ & $0.80 \pm 0.09$ \\
\hline
\end{tabular}


TABLE XI. (Continued)

\begin{tabular}{|c|c|c|c|c|}
\hline Initial state & Final state & $\Gamma_{\text {The }}(\mathrm{keV})$ & $\mathcal{B}_{\text {The }}\left(\times 10^{-2}\right)$ & $\mathcal{B}_{\operatorname{Exp}}\left(\times 10^{-2}\right)$ \\
\hline \multirow{23}{*}{$\Upsilon(11020)$} & $\pi \pi \Upsilon(2 S)$ & 17.89 & $3.25 \times 10^{-2}$ & $1.17 \pm 0.20$ \\
\hline & $\pi \pi \Upsilon(3 S)$ & 5.56 & $1.01 \times 10^{-2}$ & $0.72_{-0.26}^{+0.29}$ \\
\hline & $\pi \pi \Upsilon(4 S)$ & $8.54 \times 10^{-2}$ & $1.55 \times 10^{-4}$ & $\ldots$ \\
\hline & $e^{+} e^{-}$ & 0.15 & $1.90 \times 10^{-4}$ & $(1.6 \pm 0.5) \times 10^{-4}$ \\
\hline & $3 g$ & 11.57 & $1.46 \times 10^{-2}$ & $\ldots$ \\
\hline & $\gamma g g$ & 0.22 & $2.78 \times 10^{-4}$ & $\cdots$ \\
\hline & $3 \gamma$ & $9.56 \times 10^{-7}$ & $1.21 \times 10^{-9}$ & $\cdots$ \\
\hline & $\gamma \chi_{b 0}(1 P)$ & $4.08 \times 10^{-2}$ & $5.16 \times 10^{-5}$ & $\cdots$ \\
\hline & $\gamma \chi_{b 1}(1 P)$ & $3.32 \times 10^{-2}$ & $4.20 \times 10^{-5}$ & $\cdots$ \\
\hline & $\gamma \chi_{b 2}(1 P)$ & $8.12 \times 10^{-3}$ & $1.03 \times 10^{-5}$ & $\cdots$ \\
\hline & $\gamma \chi_{b 0}(2 P)$ & 0.11 & $1.39 \times 10^{-4}$ & $\cdots$ \\
\hline & $\gamma \chi_{b 1}(2 P)$ & 0.11 & $1.39 \times 10^{-4}$ & $\cdots$ \\
\hline & $\gamma \chi_{b 2}(2 P)$ & $5.12 \times 10^{-2}$ & $6.48 \times 10^{-5}$ & $\cdots$ \\
\hline & $\gamma \chi_{b 0}(3 P)$ & 0.26 & $3.29 \times 10^{-4}$ & $\cdots$ \\
\hline & $\gamma \chi_{b 1}(3 P)$ & 0.35 & $4.43 \times 10^{-4}$ & $\cdots$ \\
\hline & $\gamma \chi_{b 2}(3 P)$ & 0.27 & $3.42 \times 10^{-4}$ & $\cdots$ \\
\hline & $\gamma \eta_{b}(1 S)$ & $5.08 \times 10^{-2}$ & $6.43 \times 10^{-5}$ & $\cdots$ \\
\hline & $\gamma \eta_{b}(2 S)$ & $1.87 \times 10^{-2}$ & $2.37 \times 10^{-5}$ & $\cdots$ \\
\hline & $\gamma \eta_{b}(3 S)$ & $1.15 \times 10^{-2}$ & $1.46 \times 10^{-5}$ & $\cdots$ \\
\hline & $\pi \pi \Upsilon(1 S)$ & 276.20 & 0.35 & $\cdots$ \\
\hline & $\pi \pi \Upsilon(2 S)$ & 6.32 & $8.00 \times 10^{-3}$ & $\cdots$ \\
\hline & $\pi \pi \Upsilon(3 S)$ & 38.81 & $4.91 \times 10^{-2}$ & $\cdots$ \\
\hline & $\pi \pi \Upsilon(4 S)$ & 1.29 & $1.63 \times 10^{-3}$ & $\cdots$ \\
\hline
\end{tabular}

mass to conventional quarkonium states leads to large enhancements in some hadronic transition decay rates. We do not find any hybrid state around the $\Upsilon(10860)$ resonance. Therefore, other mechanism is needed in order to explain the large $\pi \pi$ decay rates observed experimentally. The authors of Ref. [84] have recently analyzed the Belle data on the cross section of the process $e^{+} e^{-} \rightarrow \Upsilon(n S) \pi \pi$ with $n=1,2$ and around the $\Upsilon(10860)$ energy region. They found that the experimental data is compatible with a tetraquark interpretation for the $\Upsilon(10860)$. Further studies are needed to understand the anomalous $\pi \pi$ decay widths of the $\Upsilon(10860)$.

Table XII shows the open-flavor strong decay widths of the $\Upsilon(4 S), \Upsilon(10860)$ and $\Upsilon(11020)$ states. We calculate these decays using a version of the ${ }^{3} P_{0}$ model in which the strength $\gamma$ of the decay interaction scales as the reduced mass of the $q \bar{q}$-pair of the decaying meson [85]. See Appendix B for further details. Following Eq. (B14), the value of $\gamma$ of the ${ }^{3} P_{0}$ model is 0.205 in the bottomonium sector. One can see that the general trend of the total decay widths is well reproduced.

The $\Upsilon(4 S)$ is the first $1^{--}$bottomonium state above the $B \bar{B}$ threshold, $10.56 \mathrm{GeV}$. This state only decays into the $B \bar{B}$ final channel. We have incorporated the isospin breaking via the experimental masses. In Table XII we compare
TABLE XII. Open $b$-flavored strong decay widths, in $\mathrm{MeV}$, and branchings, in $\%$, of the $\Upsilon(4 S), \Upsilon(10860)$ and $\Upsilon(11020)$ states. Experimental data are taken from Ref. [22].

\begin{tabular}{|c|c|c|c|c|c|}
\hline Meson & State & Channel & $\Gamma_{3} P_{0}$ & $\mathcal{B}^{{ }^{3} P_{0}}$ & $\mathcal{B}_{\text {Exp }}[22]$ \\
\hline \multirow[t]{3}{*}{$\Upsilon \mathbf{r}(4 S)$} & \multirow[t]{3}{*}{$4^{3} S_{1}$} & $B^{+} B^{-}$ & 10.41 & 50.54 & $51.3 \pm 0.6$ \\
\hline & & $B^{0} \bar{B}^{0}$ & 10.18 & 49.46 & $48.7 \pm 0.6$ \\
\hline & & $B B$ & 20.59 & 100.00 & $>96$ \\
\hline $20.5 \pm 2.5$ & \multirow{8}{*}{$5^{3} S_{1}$} & total & 20.59 & 100.00 & $\ldots$ \\
\hline \multirow[t]{7}{*}{$\Upsilon(10860)$} & & $B B$ & 6.22 & 22.29 & $5.5 \pm 1.0$ \\
\hline & & $B B^{*}$ & 11.83 & 42.41 & $13.7 \pm 1.6$ \\
\hline & & $B^{*} B^{*}$ & 0.09 & 0.32 & $38.1 \pm 3.4$ \\
\hline & & $B_{s} B_{s}$ & 0.96 & 3.45 & $0.5 \pm 0.5$ \\
\hline & & $B_{s} B_{s}^{*}$ & 1.15 & 4.11 & $1.5 \pm 0.7$ \\
\hline & & $B_{s}^{*} B_{s}^{*}$ & 7.65 & 27.42 & $17.9 \pm 2.8$ \\
\hline & & $B_{s}^{(*)} B_{s}^{(*)}$ & 9.76 & 34.98 & $19.9 \pm 3.0$ \\
\hline \multirow{9}{*}{$\begin{array}{l}55 \pm 28 \\
\Upsilon(11020)\end{array}$} & \multirow{9}{*}{$6^{3} S_{1}$} & total & 27.89 & 100.00 & $\ldots$ \\
\hline & & $B B$ & 4.18 & 5.28 & $\ldots$ \\
\hline & & $B B^{*}$ & 15.49 & 19.57 & $\ldots$ \\
\hline & & $B B_{1}$ & 40.08 & 50.64 & $\ldots$ \\
\hline & & $B B_{1}^{\prime}$ & 3.95 & 4.98 & $\ldots$ \\
\hline & & $B^{*} B^{*}$ & 11.87 & 14.99 & $\cdots$ \\
\hline & & $B_{s} B_{s}$ & 0.07 & 0.09 & $\ldots$ \\
\hline & & $B_{s} B_{s}^{*}$ & 1.50 & 1.89 & $\ldots$ \\
\hline & & $B_{s}^{*} B_{s}^{*}$ & 2.02 & 2.56 & $\cdots$ \\
\hline $79 \pm 16$ & & total & 79.16 & 100.00 & $\ldots$ \\
\hline
\end{tabular}


the theoretical branching fractions with the experimental ones for the two possible channels $B^{+} B^{-}$and $B^{0} \bar{B}^{0}$. Despite the mass of the $\Upsilon(4 S)$ is very close to the thresholds, the difference between branching fractions of both channels is negligible due to the small difference between masses of the $B^{ \pm}$and $B^{0}$.

The possible two-body final decay channels of the $\Upsilon(10860)$ are $B B, B B^{*}, B^{*} B^{*}, B_{s} B_{s} B_{s} B_{s}^{*} B_{s}^{*} B_{s}^{*}$. The ${ }^{3} P_{0}$ decay model predicts a total width and branching fractions that are compatible with the experimental data except for two cases in which the disagreement is quite strong. The decay channel $B^{*} B^{*}$ appears to be suppressed in the ${ }^{3} P_{0}$ model whereas seems to be the dominant one attending to the experimental data. This is due to the small value of the overlap integral between the wave functions in our model. On the other hand, the theoretical branching fraction $\mathcal{B}\left(\Upsilon(10860) \rightarrow B_{s}^{(*)} B_{s}^{(*)}\right)$ is roughly a factor of 2 bigger than the one measured experimentally. This is because we overestimate the rates of the $B_{s} B_{s}, B_{s} B_{s}^{*}$ and $B_{s}^{*} B_{s}^{*}$ final decay channels despite the order of magnitude is correctly given.

There is no experimental data about the open $b$-flavored strong decays of the $\Upsilon(11020)$ resonance. Only its total decay width is known experimentally [22] and the prediction of the ${ }^{3} P_{0}$ model is in very good agreement with such figure. The final decay channels $B B^{*}, B B_{1}$ and $B^{*} B^{*}$ appear to be dominant in our model. The partial widths of the remaining decay channels are an order of magnitude smaller.

\section{The $D$-wave levels}

Up to now, we have presented our theoretical results for the $S$ - and $P$-wave bottomonium states. It is of some interest to go beyond this. For instance, a key test of the nonrelativistic potential description of bottomonium is the confirmation of the predicted $D$-wave levels. For this reason we perform in this section a theoretical study of the mesons $\eta_{b 2}\left({ }^{1} D_{2}\right), \Upsilon\left({ }^{3} D_{1}\right), \Upsilon_{2}\left({ }^{3} D_{2}\right)$ and $\Upsilon_{3}\left({ }^{3} D_{3}\right)$.

Despite the $S$-wave and $P$-wave bottomonium states were first observed in the 1970s and 1980s, the triplet $\Upsilon\left(1^{3} D_{J}\right)$ has been observed recently [15] distinguishing only the $\Upsilon\left(1^{3} D_{2}\right)$ state [16]. The mass of the $\Upsilon_{2}(1 D)$ was measured to be $(10164.5 \pm 0.8 \pm 0.5) \mathrm{MeV}$. One can consider that the mass of the spin-triplet $\Upsilon\left(1^{3} D_{J}\right)$ should be around this value assuming that the relativistic corrections are small in the bottomonium sector. Our theoretical mass for this spin triplet is $10123 \mathrm{MeV}$ which is lower than the experimental data and also with respect to other theoretical predictions [48-53,56,86-88].

It is inferred from Table II that the next set of spin-triplet $D$-wave levels is expected in the range of $10419 \mathrm{MeV}$, and for the second radial excitation in the range of $10658 \mathrm{MeV}$. Contrary to the $1 D$ multiplet, these two values are in better agreement with those predicted by other quark models. The fine structure within the $D$-wave multiplets is predicted to be somewhat smaller in the present model than in those of
TABLE XIII. Decay widths and branching fractions of annihilation rates, radiative decays and hadronic transitions for the $\eta_{b 2}(1 D)$ and $\eta_{b 2}(2 D)$ states. There is no experimental data available.

\begin{tabular}{llcc}
\hline \hline Initial state & Final state & $\Gamma_{\text {The }}(\mathrm{keV})$ & $\mathcal{B}_{\text {The }}\left(\times 10^{-2}\right)$ \\
\hline$\eta_{b 2}(1 D)$ & $g g$ & 0.37 & 2.07 \\
& $\gamma h_{b}(1 P)$ & 17.23 & 96.58 \\
& $\pi \pi \eta_{b}(1 S)$ & 0.24 & 1.35 \\
$\eta_{b 2}(2 D)$ & total & 17.84 & 100.00 \\
& $g g$ & 0.67 & 3.57 \\
& $\gamma h_{b}(1 P)$ & 4.15 & 22.13 \\
& $\gamma h_{b}(2 P)$ & 11.66 & 62.18 \\
& $\gamma h_{b 3}(1 F)$ & 2.20 & 11.73 \\
& $\gamma \Upsilon_{2}(1 D)$ & $1.27 \times 10^{-4}$ & $6.77 \times 10^{-4}$ \\
& $\gamma \Upsilon_{3}(1 D)$ & $5.30 \times 10^{-6}$ & $2.83 \times 10^{-5}$ \\
& $\pi \pi \eta_{b}(1 S)$ & $6.44 \times 10^{-2}$ & 0.34 \\
& $\pi \pi \eta_{b}(2 S)$ & $8.39 \times 10^{-3}$ & $4.47 \times 10^{-2}$ \\
& total & 18.75 & 100.00 \\
\hline \hline
\end{tabular}

other authors, but there is a general agreement about these mass-splittings to be in the order of $\pm 10 \mathrm{MeV}$.

Despite the spin-singlet $D$-wave states, $\eta_{b 2}$, are expected to lie very close in mass to the spin-weighted average of the spin-triplet $D$-wave states, $\Upsilon_{J}$, they are still missing experimentally. One possibility to find the $1^{1} D_{2}$ and $2^{1} D_{2}$ states is studying the E1 radiative decays $h_{b}(2 P) \rightarrow$ $\gamma \eta_{b 2}(1 D)$ and $h_{b}(3 P) \rightarrow \gamma \eta_{b 2}(2 D)$. Our prediction for both decays is in the order of $5 \mathrm{keV}$ (see Table V). However, a better possibility is studying the decays $\eta_{b 2}(1 D) \rightarrow$ $\gamma h_{b}(1 P)$ and $\eta_{b 2}(2 D) \rightarrow \gamma h_{b}(2 P)$ because the final states are well established in the PDG and our model predicts decay rates of few tens of $\mathrm{keV}$ (see Table XIII). The decay rate of the $\eta_{b 2}(2 D) \rightarrow \gamma h_{b}(1 P)$ transition is an order of magnitude smaller than the previous ones and thus its observation seems to be complicated.

Table XIV shows the decay widths and branching fractions of annihilation rates, radiative decays and hadronic transitions for the $1 D$ and $2 D$ states of the $\Upsilon, \Upsilon_{2}$ and $\Upsilon_{3}$ mesons. As one can see in the Table, the di-electron decay rates of the $\Upsilon D$-wave states are 2 orders of magnitude smaller than those of the $S$-wave states (see Tables VIII and XI). The $\Upsilon$ family can be studied easily via $e^{+} e^{-}$annihilation as they have the same quantum numbers of the emitted virtual photon. However, the production rate in this reaction is related with the leptonic width and we have seen that they are very small for the $\Upsilon D$-wave states. This is the reason why there is no experimental confirmation of the $1^{--} D$-wave states. At this point, it is also worth remembering that the di-electron width of a $Q \bar{Q}$ meson is orders of magnitude larger than the corresponding one for a multiquark system [89].

We have mentioned above that the constant $C_{2}$ of Eq. (B29) is fixed through the transition $\Upsilon_{2}(1 D) \rightarrow$ $\pi \pi \Upsilon(1 S)$. This decay implies a $D \rightarrow S$ transition, and thus 
TABLE XIV. Decay widths and branching fractions of annihilation rates, radiative decays and hadronic transitions for the $1 D$ and $2 D$ states of the $\Upsilon, \Upsilon_{2}$ and $\Upsilon_{3}$ mesons. An estimate of the theoretical total decay width is provided. Experimental data are taken from Ref. [22].

\begin{tabular}{|c|c|c|c|c|}
\hline Initial state & Final state & $\Gamma_{\text {The }}(\mathrm{keV})$ & $\mathcal{B}_{\text {The }}\left(\times 10^{-2}\right)$ & $\mathcal{B}_{\operatorname{Exp}}[22]\left(\times 10^{-2}\right)$ \\
\hline \multirow[t]{7}{*}{$\Upsilon(1 D)$} & $e^{+} e^{-}$ & $1.40 \times 10^{-3}$ & $3.17 \times 10^{-3}$ & $\cdots$ \\
\hline & $3 g$ & 9.97 & 22.57 & $\ldots$ \\
\hline & $\gamma \chi_{b 0}(1 P)$ & 20.98 & 47.49 & $\cdots$ \\
\hline & $\gamma \chi_{b 1}(1 P)$ & 12.29 & 27.82 & $\cdots$ \\
\hline & $\gamma \chi_{b 2}(1 P)$ & 0.65 & 1.47 & $\cdots$ \\
\hline & $\pi \pi \Upsilon(1 S)$ & 0.29 & 0.66 & $<0.82[16]$ \\
\hline & total & 44.18 & 100.00 & $\ldots$ \\
\hline \multirow[t]{5}{*}{$\Upsilon_{2}(1 D)$} & $3 g$ & 0.62 & 2.13 & $\cdots$ \\
\hline & $\gamma \chi_{b 1}(1 P)$ & 21.95 & 75.46 & $\cdots$ \\
\hline & $\gamma \chi_{b 2}(1 P)$ & 6.23 & 21.42 & $\cdots$ \\
\hline & $\pi \pi \Upsilon(1 S)$ & 0.29 & 1.00 & $0.99_{-0.21}^{+0.23} \pm 0.09$ \\
\hline & total & 29.09 & 100.0 & $\ldots$ \\
\hline \multirow[t]{4}{*}{$\Upsilon_{3}(1 D)$} & $3 g$ & 0.22 & 0.87 & $\cdots$ \\
\hline & $\gamma \chi_{b 2}(1 P)$ & 24.74 & 97.98 & $\cdots$ \\
\hline & $\pi \pi \Upsilon(1 S)$ & 0.29 & 1.15 & $<0.62[16]$ \\
\hline & total & 25.25 & 100.00 & $\ldots$ \\
\hline \multirow[t]{13}{*}{$\Upsilon(2 D)$} & $e^{+} e^{-}$ & $2.50 \times 10^{-3}$ & $8.24 \times 10^{-3}$ & $\ldots$ \\
\hline & $3 g$ & 9.69 & 31.93 & $\cdots$ \\
\hline & $\gamma \chi_{b 0}(1 P)$ & 3.52 & 11.60 & $\cdots$ \\
\hline & $\gamma \chi_{b 1}(1 P)$ & 1.58 & 5.21 & $\cdots$ \\
\hline & $\gamma \chi_{b 2}(1 P)$ & $6.08 \times 10^{-2}$ & 0.20 & $\cdots$ \\
\hline & $\gamma \chi_{b 0}(2 P)$ & 8.35 & 27.52 & $\cdots$ \\
\hline & $\gamma \chi_{b 1}(2 P)$ & 4.84 & 15.95 & $\cdots$ \\
\hline & $\gamma \chi_{b 2}(2 P)$ & 0.24 & 0.79 & $\cdots$ \\
\hline & $\gamma \chi_{b 2}(1 F)$ & 2.05 & 6.76 & $\cdots$ \\
\hline & $\gamma \eta_{b 2}(1 D)$ & $4.46 \times 10^{-6}$ & $1.47 \times 10^{-5}$ & $\ldots$ \\
\hline & $\pi \pi \Upsilon(1 S)$ & $7.10 \times 10^{-3}$ & $2.34 \times 10^{-2}$ & $\cdots$ \\
\hline & $\pi \pi \Upsilon(2 S)$ & $3.98 \times 10^{-3}$ & $1.31 \times 10^{-2}$ & $\ldots$ \\
\hline & total & 30.34 & 100.00 & $\ldots$ \\
\hline \multirow[t]{11}{*}{$\Upsilon_{2}(2 D)$} & $3 g$ & 0.61 & 3.26 & $\ldots$ \\
\hline & $\gamma \chi_{b 1}(1 P)$ & 3.43 & 18.35 & $\cdots$ \\
\hline & $\gamma \chi_{b 2}(1 P)$ & 0.80 & 4.28 & $\cdots$ \\
\hline & $\gamma \chi_{b 1}(2 P)$ & 9.10 & 48.69 & $\cdots$ \\
\hline & $\gamma \chi_{b 2}(2 P)$ & 2.55 & 13.64 & $\ldots$ \\
\hline & $\gamma \chi_{b 2}(1 F)$ & 0.25 & 1.34 & $\ldots$ \\
\hline & $\gamma \chi_{b 3}(1 F)$ & 1.93 & 10.33 & $\ldots$ \\
\hline & $\gamma \eta_{b 2}(1 D)$ & $1.35 \times 10^{-4}$ & $7.22 \times 10^{-4}$ & $\ldots$ \\
\hline & $\pi \pi \Upsilon(1 S)$ & $1.54 \times 10^{-2}$ & $8.24 \times 10^{-2}$ & $\cdots$ \\
\hline & $\pi \pi \Upsilon(2 S)$ & $4.52 \times 10^{-3}$ & $2.42 \times 10^{-2}$ & $\ldots$ \\
\hline & total & 18.69 & 100.00 & $\ldots$ \\
\hline \multirow{10}{*}{$\Upsilon_{3}(2 D)$} & $3 g$ & 1.25 & 7.82 & $\ldots$ \\
\hline & $\gamma \chi_{b 2}(1 P)$ & 3.80 & 23.79 & $\cdots$ \\
\hline & $\gamma \chi_{b 2}(2 P)$ & 10.70 & 66.98 & $\cdots$ \\
\hline & $\gamma \chi_{b 2}(1 F)$ & $4.96 \times 10^{-3}$ & $3.10 \times 10^{-2}$ & $\ldots$ \\
\hline & $\gamma \chi_{b 3}(1 F)$ & 0.19 & 1.19 & $\cdots$ \\
\hline & $\gamma \eta_{b 2}(1 D)$ & $5.68 \times 10^{-4}$ & $3.56 \times 10^{-3}$ & $\cdots$ \\
\hline & $\gamma \eta_{b 2}(2 D)$ & $8.73 \times 10^{-7}$ & $5.46 \times 10^{-6}$ & $\cdots$ \\
\hline & $\pi \pi \Upsilon(1 S)$ & $2.55 \times 10^{-2}$ & 0.16 & $\cdots$ \\
\hline & $\pi \pi \Upsilon(2 S)$ & $5.13 \times 10^{-3}$ & $3.21 \times 10^{-2}$ & $\ldots$ \\
\hline & total & 15.98 & 100.00 & $\ldots$ \\
\hline
\end{tabular}


TABLE XV. Radiative decay chains involving the photon cascades $3 S \rightarrow 2 P \rightarrow 2 S \rightarrow 1 P$ and $3 S \rightarrow 2 P \rightarrow 1 D \rightarrow 1 P$. The branching fractions are $\mathcal{B}_{1}=\mathcal{B}\left(3^{3} S_{1} \rightarrow 2^{3} P_{J}+\gamma\right), \mathcal{B}_{2}=\mathcal{B}\left(2^{3} P_{J} \rightarrow 2^{3} S_{1}+\gamma\right)$ or $=\mathcal{B}\left(2^{3} P_{J} \rightarrow 1^{3} D_{J}+\gamma\right), \mathcal{B}_{3}=\mathcal{B}\left(2^{3} S_{1} \rightarrow 1^{3} P_{J}+\gamma\right)$ or $=\mathcal{B}\left(1^{3} D_{J} \rightarrow 1^{3} P_{J}+\gamma\right)$, and $\mathcal{B}_{\text {Tot }}=\mathcal{B}_{1} \times \mathcal{B}_{2} \times \mathcal{B}_{3}$.

\begin{tabular}{|c|c|c|c|c|}
\hline Decay chain & $\mathcal{B}_{1}(\%)$ & $\mathcal{B}_{2}(\%)$ & $\mathcal{B}_{3}(\%)$ & $\mathcal{B}_{\text {Tot }}\left(10^{-6}\right)$ \\
\hline $3^{3} S_{1} \rightarrow 2^{3} P_{0} \rightarrow 2^{3} S_{1} \rightarrow 1^{3} P_{0}$ & 5.96 & 0.54 & 3.41 & 10.98 \\
\hline$\rightarrow 1^{3} P_{1}$ & 5.96 & 0.54 & 5.75 & 18.51 \\
\hline$\rightarrow 1^{3} P_{2}$ & 5.96 & 0.54 & 6.50 & 20.92 \\
\hline$\rightarrow 1^{3} D_{1} \rightarrow 1^{3} P_{0}$ & 5.96 & 0.031 & 47.49 & 8.75 \\
\hline$\rightarrow 1^{3} P_{1}$ & 5.96 & 0.031 & 27.82 & 5.12 \\
\hline$\rightarrow 1^{3} P_{2}$ & 5.96 & 0.031 & 1.47 & 0.27 \\
\hline $3^{3} S_{1} \rightarrow 2^{3} P_{1} \rightarrow 2^{3} S_{1} \rightarrow 1^{3} P_{0}$ & 10.48 & 11.91 & 3.41 & 425.63 \\
\hline$\rightarrow 1^{3} P_{1}$ & 10.48 & 11.91 & 5.75 & 717.70 \\
\hline$\rightarrow 1^{3} P_{2}$ & 10.48 & 11.91 & 6.50 & 811.31 \\
\hline$\rightarrow 1^{3} D_{1} \rightarrow 1^{3} P_{0}$ & 10.48 & 0.31 & 47.49 & 154.29 \\
\hline$\rightarrow 1^{3} P_{1}$ & 10.48 & 0.31 & 27.82 & 90.38 \\
\hline$\rightarrow 1^{3} P_{2}$ & 10.48 & 0.31 & 1.47 & 4.78 \\
\hline$\rightarrow 1^{3} D_{2} \rightarrow 1^{3} P_{1}$ & 10.48 & 0.95 & 75.46 & 751.28 \\
\hline$\rightarrow 1^{3} P_{2}$ & 10.48 & 0.95 & 21.42 & 213.26 \\
\hline $3^{3} S_{1} \rightarrow 2^{3} P_{2} \rightarrow 2^{3} S_{1} \rightarrow 1^{3} P_{0}$ & 12.60 & 12.86 & 3.41 & 552.54 \\
\hline$\rightarrow 1^{3} P_{1}$ & 12.60 & 12.86 & 5.75 & 931.71 \\
\hline$\rightarrow 1^{3} P_{2}$ & 12.60 & 12.86 & 6.50 & 1053.23 \\
\hline$\rightarrow 1^{3} D_{1} \rightarrow 1^{3} P_{0}$ & 12.60 & 0.015 & 47.49 & 9.22 \\
\hline$\rightarrow 1^{3} P_{1}$ & 12.60 & 0.015 & 27.82 & 5.40 \\
\hline$\rightarrow 1^{3} P_{2}$ & 12.60 & 0.015 & 1.47 & 0.29 \\
\hline$\rightarrow 1^{3} D_{2} \rightarrow 1^{3} P_{1}$ & 12.60 & 0.26 & 75.46 & 247.21 \\
\hline$\rightarrow 1^{3} P_{2}$ & 12.60 & 0.26 & 21.42 & 70.17 \\
\hline$\rightarrow 1^{3} D_{3} \rightarrow 1^{3} P_{2}$ & 12.60 & 1.51 & 97.98 & 1864.17 \\
\hline
\end{tabular}

it is the cleanest way to determine the constant $C_{2}$. Moreover, it is the only decay of this kind that presents a measurement of its branching fraction in the PDG [22]. As one can see in Table XIV, the decay widths of the $\Upsilon(1 D) \rightarrow \pi \pi \Upsilon(1 S), \Upsilon_{2}(1 D) \rightarrow \pi \pi \Upsilon(1 S)$ and $\Upsilon_{3}(1 D) \rightarrow$ $\pi \pi \Upsilon(1 S)$ transitions are very similar, in the order of tenths of $\mathrm{keV}$. The predicted branching fractions are in reasonable good agreement with the experimental upper limits [16].

One can inferred from Table XIV that the radiative decay rates of the $1 D$ states are dominant. The $\Upsilon(1 D)$ state decays radiatively into the $\chi_{b J}(1 P)$ with $J=0,1,2$; the $\Upsilon_{2}(1 D)$ only for $J=1,2$; and the $\Upsilon_{3}(1 D)$ only for $J=2$. The partial widths of the $\Upsilon(1 D) \rightarrow \gamma \chi_{b 0}(1 P), \Upsilon_{2}(1 D) \rightarrow$ $\gamma \chi_{b 1}(1 P)$ and $\Upsilon_{3}(1 D) \rightarrow \gamma \chi_{b 2}(1 P)$ processes are the largest ones with values around $20-25 \mathrm{keV}$. An interesting feature can be deduced from here, the strength of the radiative decay into $\chi_{b J_{f}}$ final meson depends on the total spin $J_{i}$ of the initial $\Upsilon_{J_{i}}$ being of the same order of magnitude when $J_{i}=J_{f}+1$. This implies that one needs to design an experiment involving very high spin resonances in order to find simultaneously the $\Upsilon_{J}$ states in radiative decays. This would explain why the spin-triplet $1 D$ multiplet has been observed for the first time with enough significance in the $\pi \pi \Upsilon(1 S)$ final decay channel [16].

Table XIV shows that the $2 D$ states have similar decay features as the $1 D$ states: (i) they can decay into $\pi \pi \Upsilon(1 S)$ and $\pi \pi \Upsilon(2 S)$ final channels but with partial widths much smaller than those of the $1 D$ states, (ii) the radiative decays are the dominant ones but now the largest decay rate is in the order of $10 \mathrm{keV}$, and (iii) one can observe again that the strongest radiative decay into $\chi_{b J_{f}}$ is the one in which the total spin $J_{i}$ of the initial $\Upsilon_{J_{i}}$ is equal to $J_{f}+1$. The difference here is that this fact was observed for the $1 D \rightarrow$ $1 P$ transitions and now it is fulfilled by the $2 D \rightarrow 2 P$ transitions.

It is also inferred from Table XIV that the $1 D$ and $2 D$ states of the $\Upsilon, \Upsilon_{2}$ and $\Upsilon_{3}$ mesons are quite narrow with total decay widths in the order of $15-45 \mathrm{keV}$. Moreover, the decay rate of their annihilation into gluons is not relevant except for the $1 D$ and $2 D \Upsilon$ states with branching fractions of $23 \%$ and $32 \%$, respectively.

Photon cascade processes are usually used in order to study conventional bottomonium states which are located below the open $b$-flavored threshold. If we focus on the photon cascades starting from the $\Upsilon(3 S)$, the usual process is $3 S \rightarrow 2 P \rightarrow 2 S$ which is experimentally identified via the subsequent $e^{+} e^{-}$or $\mu^{+} \mu^{-}$decay of the $2 S$ state. However, if the three-photon cascade $3 S \rightarrow 2 P \rightarrow 2 S \rightarrow$ $1 P$ can be observed, there is hope for observing the corresponding $3 S \rightarrow 2 P \rightarrow 1 D \rightarrow 1 P$ and thus a new possibility of studying the $1 D$-multiplet appears. It is worth mentioning here that all the radiative decays corresponding 
to the $3 S \rightarrow 2 P \rightarrow 2 S \rightarrow 1 P$ decay chain have been measured separately. The combined branching fractions of the three-photon cascades $3 S \rightarrow 2 P \rightarrow 2 S \rightarrow 1 P$ and $3 S \rightarrow$ $2 P \rightarrow 1 D \rightarrow 1 P$ are shown in Table XV. The most prominent cascades involving $1 D$ states are

$$
\begin{array}{ll}
3^{3} S_{1} \rightarrow 2^{3} P_{1} \rightarrow 1^{3} D_{2} \rightarrow 1^{3} P_{1} & \left(\mathcal{B}=7.51 \times 10^{-4}\right), \\
3^{3} S_{1} \rightarrow 2^{3} P_{2} \rightarrow 1^{3} D_{3} \rightarrow 1^{3} P_{2} & \left(\mathcal{B}=18.64 \times 10^{-4}\right),
\end{array}
$$

followed by

$$
\begin{array}{ll}
3^{3} S_{1} \rightarrow 2^{3} P_{1} \rightarrow 1^{3} D_{1} \rightarrow 1^{3} P_{0} & \left(\mathcal{B}=1.54 \times 10^{-4}\right), \\
3^{3} S_{1} \rightarrow 2^{3} P_{1} \rightarrow 1^{3} D_{2} \rightarrow 1^{3} P_{2} & \left(\mathcal{B}=2.13 \times 10^{-4}\right), \\
3^{3} S_{1} \rightarrow 2^{3} P_{2} \rightarrow 1^{3} D_{2} \rightarrow 1^{3} P_{1} & \left(\mathcal{B}=2.47 \times 10^{-4}\right),
\end{array}
$$

and with

$$
\begin{array}{rlrl}
3^{3} S_{1} & \rightarrow 2^{3} P_{1} \rightarrow 1^{3} D_{1} \rightarrow 1^{3} P_{1} & \left(\mathcal{B}=0.90 \times 10^{-4}\right), \\
3^{3} S_{1} \rightarrow 2^{3} P_{2} \rightarrow 1^{3} D_{2} \rightarrow 1^{3} P_{2} & \left(\mathcal{B}=0.70 \times 10^{-4}\right),
\end{array}
$$

also significant. One can conclude that the range of possibilities is large enough in order to disentangle the masses of the $1 D$ spin-triplet members in the near future. The $1^{3} D_{2}$ and $1^{3} D_{3}$ states have more chances to be observed than the $1^{3} D_{1}$ in this kind of decay chain. The branching fractions associated with the $3^{3} S_{1} \rightarrow 2^{3} P_{1} \rightarrow$ $1^{3} D_{2} \rightarrow 1^{3} P_{1}$ and $3^{3} S_{1} \rightarrow 2^{3} P_{2} \rightarrow 1^{3} D_{3} \rightarrow 1^{3} P_{2}$ photon cascade processes that involve, respectively, the $1^{3} D_{2}$ and $1^{3} D_{3}$ states are 5 and 12 times larger than the most important three-photon cascade involving the $1^{3} D_{1}$ state, $3^{3} S_{1} \rightarrow 2^{3} P_{1} \rightarrow 1^{3} D_{1} \rightarrow 1^{3} P_{0}$.

A similar game can be played in order to give some insight on the most plausible photon cascades to study the spin-triplet $2 D$ states. Table XVI shows the two-photon cascades starting from the $\chi_{b J}(3 P)$. As we have mentioned above, a new structure centered at a mass of $10.5 \mathrm{GeV}$ has been interpreted as the $\chi_{b J}(3 P)$ system $[19,20]$. This structure is still below the open $b$-flavored threshold, and it should naturally decay into $2 D$ states by E1 radiative transitions; the second photon comes from the radiative decays of the $2 D$ states into the well established $\chi_{b J}(2 P)$ mesons. As one can see in Table XVI, the most prominent two-photon cascades are

$$
\begin{array}{ll}
3^{3} P_{1} \rightarrow 2^{3} D_{2} \rightarrow 2^{3} P_{1} & \left(\mathcal{B}=10.91 \times 10^{-3}\right), \\
3^{3} P_{2} \rightarrow 2^{3} D_{3} \rightarrow 2^{3} P_{2} & \left(\mathcal{B}=19.89 \times 10^{-3}\right),
\end{array}
$$

followed by
TABLE XVI. Radiative decay chains involving the photon cascades $3 P \rightarrow 2 D \rightarrow 2 P$. The branching fractions are $\mathcal{B}_{1}=\mathcal{B}\left(3^{3} P_{J} \rightarrow 2^{3} D_{J^{\prime}}+\gamma\right), \mathcal{B}_{2}=\mathcal{B}\left(2^{3} D_{J^{\prime}} \rightarrow 2^{3} P_{J^{\prime \prime}}+\gamma\right)$, and $\mathcal{B}_{\text {Tot }}=\mathcal{B}_{1} \times \mathcal{B}_{2}$.

\begin{tabular}{lcrr}
\hline \hline Decay chain & $\mathcal{B}_{1}(\%)$ & $\mathcal{B}_{2}(\%)$ & $\mathcal{B}_{\text {Tot }}\left(10^{-4}\right)$ \\
\hline $3^{3} P_{0} \rightarrow 2^{3} D_{1} \rightarrow 2^{3} P_{0}$ & 0.14 & 27.52 & 3.85 \\
$\rightarrow 2^{3} P_{1}$ & 0.14 & 15.95 & 2.23 \\
$\rightarrow 2^{3} P_{2}$ & 0.14 & 0.79 & 0.11 \\
$3^{3} P_{1} \rightarrow 2^{3} D_{1} \rightarrow 2^{3} P_{0}$ & 0.85 & 27.52 & 23.39 \\
$\rightarrow 2^{3} P_{1}$ & 0.85 & 15.95 & 13.56 \\
$\rightarrow 2^{3} P_{2}$ & 0.85 & 0.79 & 0.67 \\
$\rightarrow 2^{3} D_{2} \rightarrow 2^{3} P_{1}$ & 2.24 & 48.69 & 109.07 \\
$\rightarrow 2^{3} P_{2}$ & 2.24 & 13.64 & 30.55 \\
$3^{3} P_{2} \rightarrow 2^{3} D_{1} \rightarrow 2^{3} P_{0}$ & 0.13 & 27.52 & 3.58 \\
$\rightarrow 2^{3} P_{1}$ & 0.13 & 15.95 & 2.07 \\
$\rightarrow 2^{3} P_{2}$ & 0.13 & 0.79 & 0.10 \\
$\rightarrow 2^{3} D_{2} \rightarrow 2^{3} P_{1}$ & 0.56 & 48.69 & 27.27 \\
$\rightarrow 2^{3} P_{2}$ & 0.56 & 13.64 & 7.64 \\
$\rightarrow 2^{3} D_{3} \rightarrow 2^{3} P_{2}$ & 2.97 & 66.98 & 198.93 \\
\hline \hline
\end{tabular}

$$
\begin{array}{ll}
3^{3} P_{1} \rightarrow 2^{3} D_{2} \rightarrow 2^{3} P_{2} & \left(\mathcal{B}=3.06 \times 10^{-3}\right), \\
3^{3} P_{2} \rightarrow 2^{3} D_{2} \rightarrow 2^{3} P_{1} & \left(\mathcal{B}=2.73 \times 10^{-3}\right) .
\end{array}
$$

The two-photon cascades involving the $2^{3} D_{1}$ state present branching fractions smaller than the ones shown above. The two most important decay chains involving this state are

$$
\begin{array}{ll}
3^{3} P_{1} \rightarrow 2^{3} D_{1} \rightarrow 2^{3} P_{0} & \left(\mathcal{B}=2.34 \times 10^{-3}\right), \\
3^{3} P_{1} \rightarrow 2^{3} D_{1} \rightarrow 2^{3} P_{1} & \left(\mathcal{B}=1.36 \times 10^{-3}\right) .
\end{array}
$$

\section{SUMMARY AND CONCLUSIONS}

We have revisited the bottomonium spectrum motivated by the experimental progress in the last few years on determining new conventional and unconventional states in this sector. Our approach is a nonrelativistic constituent quark model whose model parameters are constrained by other quark sectors, from light to heavy, and thus our description of the bottomonium is, in this sense, parameter free.

The bottomonium spectrum predicted by our quark model is in a global agreement with the experimental data. Moreover, we have provided a large number of electromagnetic, strong and hadronic decays showing that our results are in reasonable agreement with the available experimental data in most cases. Among the results we describe, the following are of particular interest.

Our value for the mass of the $\eta_{b}(2 S)$ is within the mass range given by the $B A B A R$ Collaboration and slightly lower than the CLEO estimation. The hyperfine mass splitting between the singlet and triplet $2 S$ states is consistent with 
the experimental data and also with lattice QCD computations. A prediction of the $\eta_{b}(3 S)$ and its corresponding hyperfine mass splitting is provided. In order to give more insights about the better way to determine their properties experimentally, we have computed the decay widths and branching fractions of annihilation rates, radiative decays and hadronic transitions for the $\eta_{b}(1 S), \eta_{b}(2 S)$ and $\eta_{b}(3 S)$ states.

The masses predicted by our theoretical model for the $h_{b}$ states are located at the spin-weighted average of the corresponding triplet $\chi_{b J}$ states. This indicates that our hyperfine interaction is compatible with zero as should be from experimental observations. The decay widths of annihilation rates, radiative decays and hadronic transitions for the $h_{b}$ mesons have been also provided indicating that these states are narrow mesons with total decay widths of about $100 \mathrm{keV}$.

The ATLAS and D0 Collaborations have reported very recently the average mass of the $\chi_{b J}(3 P)$ multiplet. They were not able to distinguish the different members of this multiplet, only the LHCb Collaboration has provided a mass estimation for the $\chi_{b 1}(3 P)$ state which is in very good agreement with our quark model result. We predict intramultiplet splittings in the order of $\sim 10 \mathrm{MeV}$. We have calculated decay widths of hadronic, radiative and annihilation into gluons processes concerning the $\chi_{b J}(1 P, 2 P, 3 P)$. In general, our theoretical results are in agreement with the available experimental data. Special attention deserves the predicted decay properties of the $\chi_{b J}(3 P)$ states which can help experimentalists to determine the properties of the different members of the multiplet. It is possible that we are overestimating the annihilation rates into gluons for the $\chi_{b 0}(n P)$ states but we have not been able to give a definitive statement.

Our theoretical description of the $\gamma$ family has been exhaustive. We have provided masses and also a wide range of decay properties. Focusing on the radiative transitions, we have computed the branching fractions for all experimentally available decays and combine them in a way that allows us to compare with the most updated experimental study. In general, our theoretical results agree with experiment, although there are some cases in which the discrepancies are important. Focusing on the open-flavor strong decays, we achieve a global description of the partial and total decay widths with a version of the ${ }^{3} P_{0}$ model in which the strength $\gamma$ is scale dependent as a function of the reduced mass of the quark-antiquark pair of the decaying meson. Since the $\Upsilon(4 S), \Upsilon(10860)$ and $\Upsilon(11020)$ are above the $B \bar{B}$ threshold, the study of these states has to be done using more sophisticated approaches that incorporate the effect of meson-meson thresholds. This computation is beyond the scope of this work and thus we stress that the results herein for the above states have to be taken with care and leave the coupled-channels study for a future work.

We have investigated properties of the $D$-wave bottomonium levels and have made suggestions for their observation. Up to now, there is only experimental confirmation of the $1^{3} D_{2}$ state with a mass of about $10.16 \mathrm{GeV}$. According to our model, the next sets of the spin-triplet $D$-wave levels are expected in the range of 10.42 and $10.66 \mathrm{GeV}$. The mass splittings between members of the same multiplet are lower than $10 \mathrm{MeV}$. The ${ }^{1} D_{2}$ states can decay into the relatively new observed $h_{b}(1 P)$ and $h_{b}(2 P)$ states via radiative transitions. The best prospects for studying the $1^{3} D_{J}$ and $2^{3} D_{J}$ states appear to be their production via multiphoton cascades. It is worth emphasizing here that the strength of the radiative decay into the $\chi_{b J_{f}}$ final meson depends on the total spin $J_{i}$ of the initial $\Upsilon_{J_{i}}$ being the strongest one, in which $J_{i}=J_{f}+1$.

\section{ACKNOWLEDGMENTS}

J. Segovia would like to thank Nora Brambilla and Antonio Vairo for insightful comments. This work has been partially funded by Ministerio de Ciencia y Tecnología under Contract No. FPA2013-47443-C2-2P, by the Spanish Excellence Network on Hadronic Physics, Grant No. FIS2014-57026-REDT, and by the Spanish Ingenio-Consolider 2010 Program CPAN, Grant No. CSD2007-00042. J. Segovia acknowledges financial support from a postdoctoral IUFFyM contract of the Universidad de Salamanca, Spain, and from the Alexander von Humboldt Foundation. P. G. Ortega acknowledges financial support from the European Union's Marie Curie COFUND Grant No. PCOFUND-GA-2011-291783.

\section{APPENDIX A: CONSTITUENT QUARK MODEL}

We work within the framework of a constituent quark model proposed in Ref. [68] (see Refs. [90] and [91] for reviews). This model describes quite well hadron phenomenology and hadronic reactions [92-94]. Furthermore, it has been recently applied to mesons containing heavy quarks with great success, describing a wide range of physical observables which concern spectrum [70,95,96], strong reactions [85,97,98] and weak decays [99-101].

We have mentioned above that in the heavy quark sector chiral symmetry is explicitly broken and Goldstone-boson exchanges do not appear. Thus, one-gluon exchange and confinement are the only interactions remaining. The onegluon exchange potential contains central, tensor and spinorbit contributions given by 


$$
\begin{aligned}
V_{\mathrm{OGE}}^{\mathrm{C}}\left(\vec{r}_{i j}\right)= & \frac{1}{4} \alpha_{s}\left(\vec{\lambda}_{i}^{c} \cdot \vec{\lambda}_{j}^{c}\right)\left[\frac{1}{r_{i j}}-\frac{1}{6 m_{i} m_{j}}\left(\vec{\sigma}_{i} \cdot \vec{\sigma}_{j}\right) \frac{e^{-r_{i j} / r_{0}(\mu)}}{r_{i j} r_{0}^{2}(\mu)}\right], \\
V_{\mathrm{OGE}}^{\mathrm{T}}\left(\vec{r}_{i j}\right)= & -\frac{1}{16} \frac{\alpha_{s}}{m_{i} m_{j}}\left(\vec{\lambda}_{i}^{c} \cdot \vec{\lambda}_{j}^{c}\right)\left[\frac{1}{r_{i j}^{3}}-\frac{e^{-r_{i j} / r_{g}(\mu)}}{r_{i j}}\left(\frac{1}{r_{i j}^{2}}+\frac{1}{3 r_{g}^{2}(\mu)}+\frac{1}{r_{i j} r_{g}(\mu)}\right)\right] S_{i j}, \\
V_{\mathrm{OGE}}^{\mathrm{SO}}\left(\vec{r}_{i j}\right)= & -\frac{1}{16} \frac{\alpha_{s}}{m_{i}^{2} m_{j}^{2}}\left(\vec{\lambda}_{i}^{c} \cdot \vec{\lambda}_{j}^{c}\right)\left[\frac{1}{r_{i j}^{3}}-\frac{e^{-r_{i j} / r_{g}(\mu)}}{r_{i j}^{3}}\left(1+\frac{r_{i j}}{r_{g}(\mu)}\right)\right] \\
& \times\left[\left(\left(m_{i}+m_{j}\right)^{2}+2 m_{i} m_{j}\right)\left(\vec{S}_{+} \cdot \vec{L}\right)+\left(m_{j}^{2}-m_{i}^{2}\right)\left(\overrightarrow{S_{-}} \cdot \vec{L}\right)\right],
\end{aligned}
$$

where $r_{0}(\mu)=\hat{r}_{0} \frac{\mu_{n n}}{\mu_{i j}}$ and $r_{g}(\mu)=\hat{r}_{g} \frac{\mu_{n n}}{\mu_{i j}}$ are regulators which depend on $\mu_{i j}$, the reduced mass of the $q \bar{q}$ pair. The contact term of the central potential has been regularized as

$$
\delta\left(\vec{r}_{i j}\right) \sim \frac{1}{4 \pi r_{0}^{2}} \frac{e^{-r_{i j} / r_{0}}}{r_{i j}}
$$

The wide energy range needed to provide a consistent description of light, strange and heavy mesons requires an effective scale-dependent strong coupling constant. We use the frozen coupling constant [68]

$$
\alpha_{s}(\mu)=\frac{\alpha_{0}}{\ln \left(\frac{\mu^{2}+\mu_{0}^{2}}{\Lambda_{0}^{2}}\right)},
$$

in which $\mu$ is the reduced mass of the $q \bar{q}$ pair and $\alpha_{0}, \mu_{0}$ and $\Lambda_{0}$ are parameters of the model determined by a global fit to the meson spectra.

The different pieces of the confinement potential are

$$
\begin{aligned}
V_{\mathrm{CON}}^{\mathrm{C}}\left(\vec{r}_{i j}\right)= & {\left[-a_{c}\left(1-e^{-\mu_{c} r_{i j}}\right)+\Delta\right]\left(\vec{\lambda}_{i}^{c} \cdot \vec{\lambda}_{j}^{c}\right) } \\
V_{\mathrm{CON}}^{\mathrm{SO}}\left(\vec{r}_{i j}\right)= & -\left(\vec{\lambda}_{i}^{c} \cdot \vec{\lambda}_{j}^{c}\right) \frac{a_{c} \mu_{c} e^{-\mu_{c} r_{i j}}}{4 m_{i}^{2} m_{j}^{2} r_{i j}} \times\left[\left(\left(m_{i}^{2}+m_{j}^{2}\right)\left(1-2 a_{s}\right)\right.\right. \\
& \left.+4 m_{i} m_{j}\left(1-a_{s}\right)\right)\left(\vec{S}_{+} \cdot \vec{L}\right) \\
& \left.+\left(m_{j}^{2}-m_{i}^{2}\right)\left(1-2 a_{s}\right)\left(\vec{S}_{-} \cdot \vec{L}\right)\right]
\end{aligned}
$$

where $a_{s}$ controls the mixture between the scalar and vector Lorentz structures of the confinement. At short distances this potential presents a linear behavior with an effective confinement strength $\sigma=-a_{c} \mu_{c}\left(\vec{\lambda}_{i}^{c} \cdot \vec{\lambda}_{j}^{c}\right)$, while it becomes constant at large distances. This type of potential shows a threshold defined by

$$
V_{\mathrm{thr}}=\left\{-a_{c}+\Delta\right\}\left(\vec{\lambda}_{i}^{c} \cdot \vec{\lambda}_{j}^{c}\right)
$$

No $q \bar{q}$ bound states can be found for energies higher than this threshold. The system suffers a transition from a color string configuration between two static color sources into a pair of static mesons due to the breaking of the color string and the most favored decay into hadrons.

Among the different methods to solve the Schrödinger equation in order to find the quark-antiquark bound states, we use the Gaussian expansion method (GEM) [102] which provides enough accuracy and simplifies the subsequent evaluation of the decay amplitude matrix elements.

This procedure provides the radial wave function solution of the Schrödinger equation as an expansion in terms of basis functions

$$
R_{\alpha}(r)=\sum_{n=1}^{n_{\max }} c_{n}^{\alpha} \phi_{n l}^{G}(r)
$$

where $\alpha$ refers to the channel quantum numbers. The coefficients, $c_{n}^{\alpha}$, and the eigenvalue, $E$, are determined from the Rayleigh-Ritz variational principle,

$$
\sum_{n=1}^{n_{\max }}\left[\left(T_{n^{\prime} n}^{\alpha}-E N_{n^{\prime} n}^{\alpha}\right) c_{n}^{\alpha}+\sum_{\alpha^{\prime}} V_{n^{\prime} n}^{\alpha \alpha^{\prime}} c_{n}^{\alpha^{\prime}}=0\right]
$$

where $T_{n^{\prime} n}^{\alpha}, N_{n^{\prime} n}^{\alpha}$ and $V_{n^{\prime} n}^{\alpha \alpha^{\prime}}$ are the matrix elements of the kinetic energy, the normalization and the potential, respectively. $T_{n^{\prime} n}^{\alpha}$ and $N_{n^{\prime} n}^{\alpha}$ are diagonal, whereas the mixing between different channels is given by $V_{n^{\prime} n}^{\alpha \alpha^{\prime}}$.

Following Ref. [102], we employ Gaussian trial functions with ranges in geometric progression. This enables the optimization of ranges employing a small number of free parameters. Moreover, the geometric progression is dense at short distances, so that it enables the description of the dynamics mediated by short range potentials. The fast damping of the Gaussian tail does not represent an issue, since we can choose the maximal range much longer than the hadronic size.

\section{APPENDIX B: DECAYS AND REACTIONS}

\section{Radiative decays}

Electromagnetic E1 and M1 dominant multipole transitions have been studied since the early days of hadron spectroscopy because they allow access to heavy quarkonium states which are below open-flavor threshold. 
Moreover, they are interesting by themselves because they are an important tool for determining the internal charge structure of hadrons and their quantum numbers. From a theoretical point of view, electromagnetic transitions have been treated traditionally within the potential model approach. However, in the last decade, progress has been made using effective field theories [75,103]. The decay rate for E1 transitions between an initial state $n^{2 S+1} L_{J}$ and a final state $n^{\prime 2 S^{\prime}+1} L_{J^{\prime}}^{\prime}$ can be written as [35]

$$
\begin{aligned}
& \Gamma_{E 1}\left(n^{2 S+1} L_{J} \rightarrow n^{\prime 2 S^{\prime}+1} L_{J^{\prime}}^{\prime}\right) \\
& \quad=\frac{4 \alpha e_{b}^{2} k^{3}}{3}\left(2 J^{\prime}+1\right) S_{f i}^{E} \delta_{S S^{\prime}}\left|\mathcal{E}_{f i}\right|^{2} \frac{E_{f}}{M_{i}},
\end{aligned}
$$

where $k$ is the emitted photon momentum, $E_{f} / M_{i}$ is a relativistic correction with $M_{i}$ the mass of the initial state and $E_{f}$ the energy of the final state. The statistical factor, $S_{f i}^{E}$, is given by

$$
S_{f i}^{E}=\max \left(L, L^{\prime}\right)\left\{\begin{array}{ccc}
J & 1 & J^{\prime} \\
L^{\prime} & S & L
\end{array}\right\}^{2}
$$

If the full momentum dependence is retained, the overlap integral, $\mathcal{E}_{f i}$, is

$$
\mathcal{E}_{f i}=\frac{3}{k} \int_{0}^{\infty} R_{\alpha^{\prime}}(r)\left[\frac{k r}{2} j_{0}\left(\frac{k r}{2}\right)-j_{1}\left(\frac{k r}{2}\right)\right] R_{\alpha}(r) r^{2} d r,
$$

where $j_{i}(x)$ are the spherical Bessel functions of the first kind and $\alpha\left(\alpha^{\prime}\right)$ are the initial (final) meson quantum numbers.

The M1 radiative transitions can be evaluated with the following expression,

$$
\begin{aligned}
& \Gamma_{M 1}\left(n^{2 S+1} L_{J} \rightarrow n^{\prime 2 S^{\prime}+1} L_{J^{\prime}}^{\prime}\right) \\
& \quad=\frac{4 \alpha e_{b}^{2} k^{3}}{3 m_{b}^{2}}\left(2 J^{\prime}+1\right) S_{f i}^{M}\left|\mathcal{M}_{f i}\right|^{2} \frac{E_{f}}{M_{i}},
\end{aligned}
$$

where we use the same notation as in the E1 transitions but now

$$
\begin{aligned}
S_{f i}^{M}= & 6(2 S+1)\left(2 S^{\prime}+1\right) \\
& \times\left\{\begin{array}{lll}
J & 1 & J^{\prime} \\
S^{\prime} & L & S
\end{array}\right\}^{2}\left\{\begin{array}{ccc}
1 & 1 / 2 & 1 / 2 \\
1 / 2 & S^{\prime} & S
\end{array}\right\}^{2},
\end{aligned}
$$

and

$$
\mathcal{M}_{f i}=\int_{0}^{\infty} R_{\alpha^{\prime}}(r) j_{0}\left(\frac{k r}{2}\right) R_{\alpha}(r) r^{2} d r .
$$

\section{Annihilation decays}

The knowledge of annihilation decay rates is important for several reasons. First, this kind of decay allows us to test the wave function at very short range. Second, the annihilation decays into gluons and light quarks make significant contributions to the total decay widths of some bottomonium states. Third, the annihilation decays into leptons or photons can be useful for the production and identification of resonances. And fourth, leptonic decay rates can help to distinguish between conventional mesons and multiquark structures which have much smaller dielectron widths [89].

The dominant contribution to the decay of quarkonium states into lepton pairs proceed via a single virtual photon, as long as the mass of the initial meson state is sufficiently small that the contribution of a virtual $Z$ can be ignored. The leptonic width of ${ }^{3} S_{1}$ bottomonium including radiative QCD corrections is given by [104]

$$
\Gamma\left(n^{3} S_{1} \rightarrow e^{+} e^{-}\right)=\frac{4 \alpha^{2} e_{b}^{2}\left|R_{n}(0)\right|^{2}}{M_{n}^{2}}\left(1-\frac{16 \alpha_{s}}{3 \pi}\right),
$$

where $\alpha \simeq 1 / 137$ is the fine-structure constant and $e_{b}=$ $-1 / 3$ is the charge of the bottom quark in units of the electron's charge. Similarly for $D$-wave $1^{--}$bottomonium states, the leading-order decay width into $e^{+} e^{-}$is given by [105]

$$
\Gamma\left(n^{3} D_{1} \rightarrow e^{+} e^{-}\right)=\frac{25 \alpha^{2} e_{b}^{2}}{2 m_{b}^{4} M_{n}^{2}}\left|R_{n}^{\prime \prime}(0)\right|^{2} .
$$

The leading QCD correction to this expression has been calculated in Ref. [106], but we do not considered it here.

The annihilation decay rates into gluons and/or photons of the ${ }^{3} S_{1}$ bottomonium states including radiative QCD corrections are given by $[52,53]$

$$
\begin{gathered}
\Gamma\left(n^{3} S_{1} \rightarrow 3 g\right)=\frac{10\left(\pi^{2}-9\right) \alpha_{s}^{3}}{81 \pi m_{b}^{2}}\left|R_{n S}(0)\right|^{2}\left(1-\frac{4.9 \alpha_{s}}{\pi}\right), \\
\Gamma\left(n^{3} S_{1} \rightarrow \gamma g g\right)=\frac{8\left(\pi^{2}-9\right) e_{b}^{2} \alpha \alpha_{s}^{2}}{9 \pi m_{b}^{2}}\left|R_{n S}(0)\right|^{2}\left(1-\frac{7.4 \alpha_{s}}{\pi}\right), \\
\Gamma\left(n^{3} S_{1} \rightarrow 3 \gamma\right)=\frac{4\left(\pi^{2}-9\right) e_{b}^{6} \alpha^{3}}{3 \pi m_{b}^{2}}\left|R_{n S}(0)\right|^{2}\left(1-\frac{12.6 \alpha_{s}}{\pi}\right) .
\end{gathered}
$$

The authors of Ref. [107] give general expressions for singlet quarkonium decays into two gluons or two photons:

$$
\begin{aligned}
\Gamma\left(n^{1} S_{0} \rightarrow 2 g\right) & =\frac{2 \alpha_{s}^{2}}{3 m_{b}^{2}}\left|R_{n S}(0)\right|^{2}\left(1+\frac{4.4 \alpha_{s}}{\pi}\right), \\
\Gamma\left(n^{1} S_{0} \rightarrow 2 \gamma\right) & =\frac{3 e_{b}^{4} \alpha^{2}}{m_{b}^{2}}\left|R_{n S}(0)\right|^{2}\left(1-\frac{3.4 \alpha_{s}}{\pi}\right) .
\end{aligned}
$$


The annihilation decay widths of the $P$-wave bottomonium states depend on the derivative of the radial wave function at the origin. The relevant expressions have been summarize in Refs. [52,53] and are given here for completeness:

$\Gamma\left(n^{3} P_{0} \rightarrow 2 \gamma\right)=\frac{27 e_{b}^{4} \alpha^{2}}{m_{b}^{4}}\left|R_{n P}^{\prime}(0)\right|^{2}\left(1+\frac{0.2 \alpha_{s}}{\pi}\right)$,

$\Gamma\left(n^{3} P_{2} \rightarrow 2 \gamma\right)=\frac{36 e_{b}^{4} \alpha^{2}}{5 m_{b}^{4}}\left|R_{n P}^{\prime}(0)\right|^{2}\left(1-\frac{16 \alpha_{s}}{3 \pi}\right)$,

for their annihilation into photons, and

$$
\begin{aligned}
\Gamma\left(n^{3} P_{0} \rightarrow 2 g\right) & =\frac{6 \alpha_{s}^{2}}{m_{b}^{4}}\left|R_{n P}^{\prime}(0)\right|^{2}, \\
\Gamma\left(n^{3} P_{2} \rightarrow 2 g\right) & =\frac{8 \alpha_{s}^{2}}{5 m_{b}^{4}}\left|R_{n P}^{\prime}(0)\right|^{2}, \\
\Gamma\left(n^{3} P_{1} \rightarrow q \bar{q}+g\right) & =\frac{8 n_{f} \alpha_{s}^{3}}{9 \pi m_{b}^{4}}\left|R_{n P}^{\prime}(0)\right|^{2} \ln \left(m_{b}\langle r\rangle\right), \\
\Gamma\left(n^{1} P_{1} \rightarrow 3 g\right) & =\frac{20 \alpha_{s}^{3}}{9 \pi m_{b}^{4}}\left|R_{n P}^{\prime}(0)\right|^{2} \ln \left(m_{b}\langle r\rangle\right),
\end{aligned}
$$

for their annihilation into gluons and light quarks. We do not take into account the QCD corrections since these depend on each state and they are not known for the higher excited states. Moreover, one expects that these corrections are small as they concern to the bottomonium spectrum.

The decay rates for $\Upsilon\left({ }^{3} D_{J}\right) \rightarrow 3 g$ are dominated to leading order in logarithms by processes in which one of the three gluons is soft. (Two gluons cannot be emitted by a ${ }^{3} D_{J}$ state since the charge-conjugation eigenvalue of a ${ }^{3} D_{J}$ state is odd). The resulting expressions for the decay widths are [108]

$$
\begin{aligned}
\Gamma\left(n^{1} D_{2} \rightarrow 2 g\right) & =\frac{2 \alpha_{s}^{2}}{3 \pi m_{b}^{6}}\left|R_{n D}^{\prime \prime}(0)\right|^{2}, \\
\Gamma\left(n^{3} D_{1} \rightarrow 3 g\right) & =\frac{760 \alpha_{s}^{3}}{81 \pi m_{b}^{6}}\left|R_{n D}^{\prime \prime}(0)\right|^{2} \ln \left(4 m_{b}\langle r\rangle\right), \\
\Gamma\left(n^{3} D_{2} \rightarrow 3 g\right) & =\frac{10 \alpha_{s}^{3}}{9 \pi m_{b}^{6}}\left|R_{n D}^{\prime \prime}(0)\right|^{2} \ln \left(4 m_{b}\langle r\rangle\right), \\
\Gamma\left(n^{3} D_{3} \rightarrow 3 g\right) & =\frac{40 \alpha_{s}^{3}}{9 \pi m_{b}^{6}}\left|R_{n D}^{\prime \prime}(0)\right|^{2} \ln \left(4 m_{b}\langle r\rangle\right) .
\end{aligned}
$$

It is important to remark here that these formulas should be regarded as estimates of the partial widths for these annihilation processes rather than precise predictions. This is because considerable uncertainties arise in these expressions from the model dependence of the wave functions and possible relativistic and QCD radiative corrections.
Finally, an important progress has been done within pNRQCD in the computation of the inclusive decay widths into light hadrons, photons and lepton pairs for $S$ - and $P$ wave heavy quarkonium states [109,110] (see also Ref. [111] for a review). These expressions need six nonperturbative universal parameters plus the knowledge of the heavy quarkonium wave functions (and their derivatives) at the origin. All these unknown terms should be fixed by experiment or be computed in lattice QCD in order to avoid model dependences.

\section{Open-flavor meson strong decays}

Meson strong decay is a complex nonperturbative process that has not yet been described from first principles of QCD. Several phenomenological models have been developed to deal with this topic (see, for instance, Ref. [98] for a recent development). The most popular is the ${ }^{3} P_{0}$ model [61-63] which assumes that a quarkantiquark pair is created with vacuum quantum numbers, $J^{P C}=0^{++}$.

An important characteristic of the ${ }^{3} P_{0}$ model, apart from its simplicity, is that it provides the gross features of various transitions with only one parameter, the strength $\gamma$ of the decay interaction. Some attempts have been done to find possible dependences of the vertex parameter $\gamma$, see [59] and references therein. In Ref. [85] we performed a global fit to the decay widths of the mesons which belong to charmed, charmed-strange, hidden charm and hidden bottom sectors and elucidated the dependence on the mass scale of the ${ }^{3} P_{0}$ free parameter $\gamma$. Further details about the global fit can be found in Ref. [85]. The running of the strength $\gamma$ of the ${ }^{3} P_{0}$ decay model is given by

$$
\gamma(\mu)=\frac{\gamma_{0}}{\log \left(\frac{\mu}{\mu_{0}}\right)},
$$

where $\mu$ is the reduced mass of the quark-antiquark in the decaying meson and, $\gamma_{0}=0.81 \pm 0.02$ and $\mu_{0}=$ $(49.84 \pm 2.58) \mathrm{MeV}$ are parameters determined by the global fit.

We get a quite reasonable global description of the total decay widths in all meson sectors, from light to heavy. All the wave functions for the mesons involved in the open-flavor strong decays are the solutions of the Schrödinger equation with the potential model described above and using the Gaussian expansion method [102]. We use when possible experimental masses of the mesons involved in the open-flavor strong decays. This is a standard procedure within the quark model approach and allows one to ensure correct phase-space of the transition. Details of the resulting matrix elements for different cases are given in Ref. [112], here we proceed to explain briefly the main ingredients in which the model is based. 


\section{a. Transition operator}

The interaction Hamiltonian involving Dirac quark fields that describes the production process is given by

$$
H_{I}=\sqrt{3} g_{s} \int d^{3} x \bar{\psi}(\vec{x}) \psi(\vec{x})
$$

where we have introduced for convenience the numerical factor $\sqrt{3}$, which will be canceled with the color factor.

If we write the Dirac fields in second quantization and keep only the contribution of the interaction Hamiltonian which creates a $(\mu \nu)$ quark-antiquark pair, we arrive, after a nonrelativistic reduction, to the following expression for the transition operator,

$$
\begin{aligned}
T= & -\sqrt{3} \sum_{\mu, \nu} \int d^{3} p_{\mu} d^{3} p_{\nu} \delta^{(3)}\left(\vec{p}_{\mu}+\vec{p}_{\nu}\right) \frac{g_{s}}{2 m_{\mu}} \sqrt{2^{5} \pi} \\
& \times\left[\mathcal{Y}_{1}\left(\frac{\vec{p}_{\mu}-\vec{p}_{\nu}}{2}\right) \otimes\left(\frac{1}{2} \frac{1}{2}\right) 1\right]_{0} a_{\mu}^{\dagger}\left(\vec{p}_{\mu}\right) b_{\nu}^{\dagger}\left(\vec{p}_{\nu}\right),
\end{aligned}
$$

where $\mu(\nu)$ are the spin, flavor and color quantum numbers of the created quark (antiquark). The spin of the quark and antiquark is coupled to one. The $\mathcal{Y}_{l m}(\vec{p})=p^{l} Y_{l m}(\hat{p})$ is the solid harmonic defined in function of the spherical harmonic.

As in Ref. [67], we fix the relation of $g_{s}$ with the dimensionless constant giving the strength of the quarkantiquark pair creation from the vacuum as $\gamma=g_{s} / 2 m$, being $m$ the mass of the created quark (antiquark). In this convention, values of the scale-dependent strength $\gamma$ in the different quark sectors following Eq. (B14) can be found in Ref. [85].

\section{b. Transition amplitude}

We are interested on the transition amplitude for the reaction $(\alpha \beta)_{A} \rightarrow(\delta \epsilon)_{B}+(\lambda \rho)_{C}$. The meson $A$ is formed by a quark $\alpha$ and antiquark $\beta$. At some point it is created a $(\mu \nu)$ quark-antiquark pair. The created $(\mu \nu)$ pair together with the $(\alpha \beta)$ pair in the original meson regroups in the two outgoing mesons via a quark rearrangement process. These final mesons are meson $B$ which is formed by the quark-antiquark pair $(\delta \epsilon)$ and meson $C$ with $(\lambda \rho)$ quarkantiquark pair.

We work in the center-of-mass reference system of meson $A$, thus we have $\vec{K}_{A}=\vec{K}_{0}=0$ with $\vec{K}_{A}$ and $\vec{K}_{0}$ the total momentum of meson $A$ and of the system $B C$ with respect to a given reference system. We can factorize the matrix element as follows:

$$
\langle B C|T| A\rangle=\delta^{(3)}\left(\vec{K}_{0}\right) \mathcal{M}_{A \rightarrow B C} .
$$

The initial state in second quantization is

$$
|A\rangle=\int d^{3} p_{\alpha} d^{3} p_{\beta} \delta^{(3)}\left(\vec{K}_{A}-\vec{P}_{A}\right) \phi_{A}\left(\vec{p}_{A}\right) a_{\alpha}^{\dagger}\left(\vec{p}_{\alpha}\right) b_{\beta}^{\dagger}\left(\vec{p}_{\beta}\right)|0\rangle,
$$

where $\alpha(\beta)$ are the spin, flavor and color quantum numbers of the quark (antiquark). The wave function $\phi_{A}\left(\vec{p}_{A}\right)$ denotes a meson $A$ in a color singlet with an isospin $I_{A}$ with projection $M_{I_{A}}$, a total angular momentum $J_{A}$ with projection $M_{A}, J_{A}$ is the coupling of angular momentum $L_{A}$ and spin $S_{A}$. The $\vec{p}_{\alpha}$ and $\vec{p}_{\beta}$ are the momentum of quark and antiquark, respectively. The $\vec{P}_{A}$ and $\vec{p}_{A}$ are the total and relative momentum of the $(\alpha \beta)$ quark-antiquark pair within the meson $A$. The final state is more complicated than the initial one because it is a two-meson state. It can be written as

$$
\begin{aligned}
|B C\rangle= & \frac{1}{\sqrt{1+\delta_{B C}}} \int d^{3} K_{B} d^{3} K_{C} \sum_{m, M_{B C}}\left\langle J_{B C} M_{B C} l m \mid J_{T} M_{T}\right\rangle \delta^{(3)}\left(\vec{K}-\vec{K}_{0}\right) \delta\left(k-k_{0}\right) \frac{Y_{l m}(\hat{k})}{k} \sum_{M_{B}, M_{C}, M_{I_{B}}, M_{I_{C}}} \\
& \times\left\langle J_{B} M_{B} J_{C} M_{C} \mid J_{B C} M_{B C}\right\rangle\left\langle I_{B} M_{I_{B}} I_{C} M_{I_{C}} \mid I_{A} M_{I_{A}}\right\rangle \int d^{3} p_{\delta} d^{3} p_{\epsilon} d^{3} p_{\lambda} d^{3} p_{\rho} \delta^{(3)}\left(\vec{K}_{B}-\vec{P}_{B}\right) \delta^{(3)}\left(\vec{K}_{C}-\vec{P}_{C}\right) \\
& \times \phi_{B}\left(\vec{p}_{B}\right) \phi_{C}\left(\vec{p}_{C}\right) a_{\delta}^{\dagger}\left(\vec{p}_{\delta}\right) b_{\epsilon}^{\dagger}\left(\vec{p}_{\epsilon}\right) a_{\lambda}^{\dagger}\left(\vec{p}_{\lambda}\right) b_{\rho}^{\dagger}\left(\vec{p}_{\rho}\right)|0\rangle,
\end{aligned}
$$

where we have followed the notation of meson $A$ for the mesons $B$ and $C$. We assume that the final state of mesons $B$ and $C$ is a spherical wave with angular momentum $l$. The relative and total momentum of mesons $B$ and $C$ are $\vec{k}_{0}$ and $\vec{K}_{0}$. The total spin $J_{B C}$ is obtained coupling the total angular momentum of mesons $B$ and $C$, and $J_{T}$ is the coupling of $J_{B C}$ and $l$.

The ${ }^{3} P_{0}$ model takes into account only diagrams in which the $(\mu \nu)$ quark-antiquark pair separates into different final mesons. This was originally motivated by the experiment and it is known as the Okubo-Zweig-Iizuka (OZI) rule [113-115] which tells us that the disconnected diagrams are more suppressed than the connected ones. The diagrams that can contribute to the decay width through the ${ }^{3} P_{0}$ model are shown in Fig. 1 .

\section{c. Decay width}

The total width is the sum over the partial widths characterized by the quantum numbers $J_{B C}$ and $l$ 

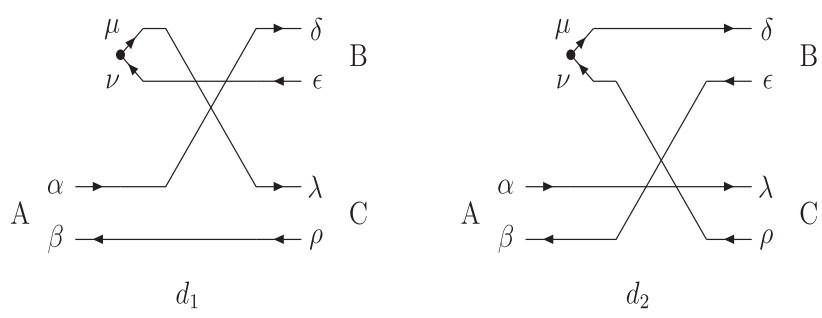

FIG. 1. Diagrams that can contribute to the decay width through the ${ }^{3} P_{0}$ model.

$$
\Gamma_{A \rightarrow B C}=\sum_{J_{B C}, l} \Gamma_{A \rightarrow B C}\left(J_{B C}, l\right)
$$

where

$\Gamma_{A \rightarrow B C}\left(J_{B C}, l\right)=2 \pi \int d k_{0} \delta\left(E_{A}-E_{B C}\right)\left|\mathcal{M}_{A \rightarrow B C}\left(k_{0}\right)\right|^{2}$.

We use relativistic phase space, so

$\Gamma_{A \rightarrow B C}\left(J_{B C}, l\right)=2 \pi \frac{E_{B}\left(k_{0}\right) E_{C}\left(k_{0}\right)}{m_{A} k_{0}}\left|\mathcal{M}_{A \rightarrow B C}\left(k_{0}\right)\right|^{2}$,

where

$k_{0}=\frac{\sqrt{\left[m_{A}^{2}-\left(m_{B}-m_{C}\right)^{2}\right]\left[m_{A}^{2}-\left(m_{B}+m_{C}\right)^{2}\right]}}{2 m_{A}}$,

is the on-shell relative momentum of mesons $B$ and $C$.

\section{Hadronic decays}

The general way of referring to an hadronic transition is [38]

$$
\Phi_{I} \rightarrow \Phi_{F}+h,
$$

where $\Phi_{I}$ and $\Phi_{F}$ stand, respectively, for the initial and final states of heavy quarkonium. The light hadron(s), $h$, are converted from emitted gluons and are kinematically dominated by single particle $\left(\pi^{0}, \eta, \omega, \ldots\right)$ or two particle $(2 \pi, 2 K, \ldots)$ states.

Since the energy difference between the initial and final quarkonium states is usually small, the emitted gluons are rather soft. In Ref. [116], Gottfried pointed out that this gluon radiation can be treated in a multipole expansion since the wavelengths of the emitted gluons are large compared with the size of the heavy quarkonium states. After the expansion of the gluon field, the Hamiltonian of the system can be decomposed as follows,

$$
\mathcal{H}_{\mathrm{QCD}}^{\mathrm{eff}}=\mathcal{H}_{\mathrm{QCD}}^{(0)}+\mathcal{H}_{\mathrm{QCD}}^{(1)}+\mathcal{H}_{\mathrm{QCD}}^{(2)},
$$

with $\mathcal{H}_{\mathrm{OCD}}^{(0)}$ the sum of the kinetic and potential energies of the heavy quarkonium, and $\mathcal{H}_{\mathrm{QCD}}^{(1)}$ and $\mathcal{H}_{\mathrm{QCD}}^{(2)}$ defined by

$$
\begin{aligned}
& \mathcal{H}_{\mathrm{QCD}}^{(1)}=Q_{a} A_{0}^{a}(x, t), \\
& \mathcal{H}_{\mathrm{QCD}}^{(2)}=-d_{a} E^{a}(x, t)-m_{a} B^{a}(x, t),
\end{aligned}
$$

in which $Q_{a}, d_{a}$ and $m_{a}$ are the color charge, the color electric dipole moment and the color magnetic dipole moment, respectively. As the $Q \bar{Q}$ pair is a color singlet, there is no contribution of the $\mathcal{H}_{\mathrm{QCD}}^{(1)}$ and only $E_{l}$ and $B_{m}$ transitions can take place.

The multipole expansion within QCD (QCDME) has been studied by many authors [116-121], but Tung-Mow Yan was the first one to present a gauge-invariant formulation in Refs. [122,123]. We will follow the updated review [124] and references therein to calculate the hadronic transitions in which we are interested. A brief description of the formulae can be found below.

\section{a. Spin-nonflip $\pi \pi$ and $\boldsymbol{\eta}$ transitions}

The spin-nonflip $\pi \pi$ decays in heavy quarkonia are dominated by double electric-dipole transitions (E1E1). Therefore, the transition amplitude can be written as follows [124],

$\mathcal{M}_{E 1 E 1}=i \frac{g_{E}^{2}}{6}\left\langle\Phi_{F} h\left|\vec{x} \cdot \vec{E} \frac{1}{E_{I}-H_{\mathrm{QCD}}^{(0)}-i D_{0}} \vec{x} \cdot \vec{E}\right| \Phi_{I}\right\rangle$,

where $\vec{x}$ is the separation between $Q$ and $\bar{Q}$, and $\left(D_{0}\right)_{b c} \equiv \delta_{b c} \partial_{0}-g_{s} f_{a b c} A_{0}^{a}$.

Inserting a complete set of intermediate states the transition amplitude (B27) becomes

$\mathcal{M}_{E 1 E 1}=i \frac{g_{E}^{2}}{6} \sum_{K L} \frac{\left\langle\Phi_{F}\left|x_{k}\right| K L\right\rangle\left\langle K L\left|x_{l}\right| \Phi_{I}\right\rangle}{E_{I}-E_{K L}}\left\langle\pi \pi\left|E_{k}^{a} E_{l}^{a}\right| 0\right\rangle$,

where $E_{K L}$ is the energy eigenvalue of the intermediate state $|K L\rangle$ with the principal quantum number $K$ and the orbital angular momentum $L$.

The intermediate states in the hadronic transition are those produced after the emission of the first gluon and before the emission of the second one. They are color singlet states with a gluon and a color-octet $Q \bar{Q}$ pair and thus these states are the so-called hybrid mesons. It is difficult to calculate these hybrid states from first principles of QCD and thus we take a reasonable model which will be explained below.

One can see in Eq. (B28) that the transition amplitude splits into two factors. The first one concerns to the wave functions and energies of the initial and final 
quarkonium states as well as those of the intermediate hybrid mesons. All these quantities can be calculated using suitable quark models. The second one describes the conversion of the emitted gluons into light hadrons. As the momenta involved are very low this matrix element cannot be calculated using perturbative QCD and one needs to resort to a phenomenological approach based on soft-pion techniques [125]. In the center-ofmass frame, the two pion momenta $q_{1}$ and $q_{2}$ are the only independent variables describing this matrix element which, in the nonrelativistic limit, can be parametrized as [122-125]

$$
\frac{g_{E}^{2}}{6}\left\langle\pi_{\alpha}\left(q_{1}\right) \pi_{\beta}\left(q_{2}\right)\left|E_{k}^{a} E_{l}^{a}\right| 0\right\rangle=\frac{\delta_{\alpha \beta}}{\sqrt{\left(2 \omega_{1}\right)\left(2 \omega_{2}\right)}}\left[C_{1} \delta_{k l} q_{1}^{\mu} q_{2 \mu}+C_{2}\left(q_{1 k} q_{2 l}+q_{1 l} q_{2 k}-\frac{2}{3} \delta_{k l} \vec{q}_{1} \cdot \vec{q}_{2}\right)\right],
$$

where $C_{1}$ and $C_{2}$ are two unknown constants. The $C_{1}$ term is isotropic, while the $C_{2}$ term has a $L=2$ angular dependence. Thus, $C_{1}$ only contributes to the $S$-wave into $S$-wave transitions and we fix it through the $\Upsilon(2 S) \rightarrow \Upsilon(1 S) \pi \pi$ reaction. The $C_{2}$ parameter is fixed through the decay $\Upsilon_{2}(1 D) \rightarrow \Upsilon(1 S) \pi \pi$.

Finally, the transition rate is given by [123]

$$
\begin{aligned}
\Gamma\left(\Phi_{I}\left({ }^{2 s+1} l_{I J_{I}}\right) \rightarrow \Phi_{F}\left({ }^{2 s+1} l_{F J_{F}}\right)+\pi \pi\right)= & \delta_{l_{I} l_{F}} \delta_{J_{I} J_{F}}\left(G\left|C_{1}\right|^{2}-\frac{2}{3} H\left|C_{2}\right|^{2}\right)\left|\sum_{L}(2 L+1)\left(\begin{array}{ccc}
l_{I} & 1 & L \\
0 & 0 & 0
\end{array}\right)\left(\begin{array}{ccc}
L & 1 & l_{I} \\
0 & 0 & 0
\end{array}\right) f_{I F}^{L 11}\right|^{2} \\
& +\left(2 l_{I}+1\right)\left(2 l_{F}+1\right)\left(2 J_{F}+1\right) \sum_{k}(2 k+1)\left(1+(-1)^{k}\right)\left\{\begin{array}{ccc}
s & l_{F} & J_{F} \\
k & J_{I} & l_{I}
\end{array}\right\}^{2} H\left|C_{2}\right|^{2} \\
& \times\left|\sum_{L}(2 L+1)\left(\begin{array}{ccc}
l_{F} & 1 & L \\
0 & 0 & 0
\end{array}\right)\left(\begin{array}{ccc}
L & 1 & l_{I} \\
0 & 0 & 0
\end{array}\right)\left\{\begin{array}{ccc}
l_{I} & L & 1 \\
1 & k & l_{F}
\end{array}\right\} f_{I F}^{L 11}\right|^{2}, \quad(\mathrm{~B} 30)
\end{aligned}
$$

with

$$
f_{I F}^{L P_{I} P_{F}}=\sum_{K} \frac{1}{M_{I}-M_{K L}}\left[\int d r r^{2+P_{F}} R_{F}(r) R_{K L}(r)\right]\left[\int d r^{\prime} r^{\prime 2+P_{I}} R_{K L}\left(r^{\prime}\right) R_{I}\left(r^{\prime}\right)\right],
$$

where $R_{I}(r)$ and $R_{F}(r)$ are, respectively, the radial wave functions of the initial and final states. $R_{K L}(r)$ is the radial wave function of the intermediate vibrational states $|K L\rangle$. The mass of the decaying meson is $M_{I}$, whereas the ones corresponding to the hybrid states are $M_{K L}$. The quantities $G$ and $H$ are the phase-space integrals,

$$
\begin{aligned}
& G=\frac{3}{4} \frac{M_{F}}{M_{I}} \pi^{3} \int d M_{\pi \pi}^{2} K\left(1-\frac{4 m_{\pi}^{2}}{M_{\pi \pi}^{2}}\right)^{1 / 2}\left(M_{\pi \pi}^{2}-2 m_{\pi}^{2}\right)^{2}, \\
& H=\frac{1}{20} \frac{M_{F}}{M_{I}} \pi^{3} \int d M_{\pi \pi}^{2} K\left(1-\frac{4 m_{\pi}^{2}}{M_{\pi \pi}^{2}}\right)^{1 / 2}\left[\left(M_{\pi \pi}^{2}-4 m_{\pi}^{2}\right)^{2}\left(1+\frac{2}{3} \frac{K^{2}}{M_{\pi \pi}^{2}}\right)+\frac{8 K^{4}}{15 M_{\pi \pi}^{4}}\left(M_{\pi \pi}^{4}+2 m_{\pi}^{2} M_{\pi \pi}^{2}+6 m_{\pi}^{4}\right)\right],
\end{aligned}
$$

with $K$ given by

$K=\frac{\sqrt{\left[\left(M_{I}+M_{F}\right)^{2}-M_{\pi \pi}^{2}\right]\left[\left(M_{I}-M_{F}\right)^{2}-M_{\pi \pi}^{2}\right]}}{2 M_{I}}$.

The leading multipoles of spin-nonflip $\eta$ transitions between spin-triplet $S$-wave states are M1M1 and E1M2. Therefore, the matrix element is given schematically by

$$
\mathcal{M}\left({ }^{3} S_{1} \rightarrow{ }^{3} S_{1}+\eta\right)=\mathcal{M}_{M 1 M 1}+\mathcal{M}_{E 1 M 2} .
$$

After some algebra and assuming that $\mathcal{M}_{M 1 M 1}=0$ (see Ref. [123] for details), the decay rate can be written as

$$
\Gamma\left(\Phi_{I}\left({ }^{3} S_{1}\right) \rightarrow \Phi_{F}\left({ }^{3} S_{1}\right)+\eta\right)=\frac{8 \pi^{2}}{27} \frac{M_{f} C_{3}^{2}}{M_{i} m_{Q}^{2}}\left|f_{I F}^{111}\right|^{2}|\vec{q}|^{3},
$$

where $\vec{q}$ is the momentum of $\eta, C_{3}$ is a new parameter which should be fixed through the $\Upsilon(2 S) \rightarrow \Upsilon(1 S) \eta$ reaction. The function $f_{I F}^{111}$ is defined in Eq. (B31).

\section{b. Spin-flip $\pi \pi$ and $\eta$ transitions}

The spin-flip $\pi \pi$ and $\eta$ transitions between heavy quarkonia are induced by an E1M1 multipole amplitude. Within the hadronization approach presented above, the 
description of this kind of decay implies the introduction of another phenomenological constant which should be fixed by experiment. Therefore, as one can deduce, the decay model for hadronic transitions begins to loose its predictive power.

In order to avoid this undesirable feature, the term which describes the conversion of the emitted gluons into light hadrons can be computed assuming a duality argument between the physical light hadron final state and the associated two-gluon final state [123]:

$$
\begin{aligned}
\Gamma\left(\Phi_{I} \rightarrow \Phi_{F}+\pi \pi\right) & \sim \Gamma\left(\Phi_{I} \rightarrow \Phi_{F} g g\right), \\
\Gamma\left(\Phi_{I} \rightarrow \Phi_{F}+\eta\right) & \sim \Gamma\left(\Phi_{I} \rightarrow \Phi_{F}(g g)_{0^{-}}\right),
\end{aligned}
$$

where in the second line the two gluons are projected into a $J^{P}=0^{-}$state to simulate the $\eta$ meson. The advantage of this approach is that we have now only two free parameters, $g_{E}$ and $g_{M}$, in order to fix the spin-nonflip and spin-flip $\pi \pi$ and $\eta$ hadronic transitions.

Explicit expressions within this new approach of the decay rates for the spin-nonflip $\pi \pi$ and $\eta$ transitions can be found in Refs. [123,124]. The decay rates for the spin-flip $\pi \pi$ and $\eta$ transitions are

$$
\begin{aligned}
\Gamma\left(\Phi_{I}\left({ }^{3} l_{I J_{I}}\right) \rightarrow \Phi_{F}\left({ }^{1} l_{F J_{F}}\right)+\pi \pi\right) \\
=\left(\frac{g_{E} g_{M}}{6 m_{Q}}\right)^{2} \frac{\left(M_{i}-M_{f}\right)^{7}}{315 \pi^{3}}\left(2 l_{F}+1\right) \\
\quad \times\left(\begin{array}{ccc}
l_{F} & 1 & l_{I} \\
0 & 0 & 0
\end{array}\right)^{2}\left|f_{I F}^{l_{F} 10}+f_{I F}^{l_{I} 01}\right|^{2}, \\
\Gamma\left(\Phi_{I}\left({ }^{3} S_{J_{I}}\right) \rightarrow \Phi_{F}\left({ }^{1} P_{J_{F}}\right)+\eta\right) \\
=\frac{g_{M}^{2}}{g_{E}^{2}} \frac{E_{F}}{M_{I}}|\vec{q}| \frac{\pi}{1144 m_{Q}^{2}}\left(\frac{4 \pi}{\sqrt{6}} f_{\pi} m_{\eta}^{2}\right)^{2}\left|f_{I F}^{110}+f_{I F}^{001}\right|^{2} .
\end{aligned}
$$

The decay rate of the spin-flip $\eta$ transition in Eq. (B37) can be read from the decay rate of the isospin violating hadronic transition [124]

$$
\begin{aligned}
\Gamma\left(\Phi_{I}\left({ }^{3} S_{J_{I}}\right) \rightarrow \Phi_{F}\left({ }^{1} P_{J_{F}}\right)+\pi^{0}\right) \\
=\frac{g_{M}^{2}}{g_{E}^{2}} \frac{E_{F}}{M_{I}}|\vec{q}| \frac{\pi}{1144 m_{Q}^{2}}\left(\frac{4 \pi}{\sqrt{2}} \frac{m_{d}-m_{u}}{m_{d}+m_{u}} f_{\pi} m_{\pi}^{2}\right)^{2} \\
\quad \times\left|f_{I F}^{110}+f_{I F}^{001}\right|^{2},
\end{aligned}
$$

in which the factor $\left(m_{d}-m_{u}\right) /\left(m_{d}+m_{u}\right)$ reflects the violation of isospin.

\section{c. A model for hybrid mesons}

From the generic properties of QCD, we might expect to have states in which the gluonic field itself is excited and carries $J^{P C}$ quantum numbers. A bound-state is called glueball when any valence quark content is absent, the addition of a constituent quark-antiquark pair to an excited gluonic field gives rise to what is called a hybrid meson. The gluonic quantum numbers couple to those of the $q \bar{q}$ pair. This coupling may give rise to the so-called exotic $J^{P C}$ mesons, but also can produce hybrid mesons with natural quantum numbers. We are interested on the last ones because they are involved in the calculation of hadronic transitions within the QCDME approach.

Ab-initio QCD calculations of the hybrid (even conventional) excited bottomonium states are particularly difficult because the large mass of the $b$-quark. For instance, full lattice QCD results can be found only for the charmonium hybrids in Ref. [126] and the first application of the effective field theory pNRQCD to the hybrid meson spectrum has been published very recently in Ref. [127].

An extension of the quark model described above to include hybrid states has been presented in Ref. [82]. This extension is inspired on the Buchmuller-Tye quarkconfining string (QCS) model [128-130]. The QCS model is defined by a relativistic-, gauge- and reparametrizationinvariant action describing quarks interacting with color $S U(3)$ gauge fields in a two-dimensional world sheet. It is assumed that the meson is composed of a quark and antiquark linked by an appropriate color electric flux line (the string).

The string can carry energy-momentum only in the region between the quark and the antiquark. The string and the quark-antiquark pair can rotate as a unit and also vibrate. Ignoring its vibrational motion, the equation which describes the dynamics of the quark-antiquark pair linked by the string should be the usual Schrödinger equation with a confinement potential. Gluon excitation effects are described by the vibration of the string. These vibrational modes provide new states beyond the naive meson picture.

A complete description of the model can be found in Refs. [128-130]. We will give here only a brief description of it. The dynamics of the string is defined by the action

$$
\begin{aligned}
S= & \int_{-\infty}^{\infty} d^{2} u \sqrt{-g} \\
& \times\left\{\sum_{j} \bar{\psi}_{j}\left[\gamma_{\mu} \tau^{\alpha \mu}\left(\frac{i}{2} \bar{\partial}_{\alpha}-e B_{a \alpha} T^{\alpha}\right)-M_{j}\right] \psi_{j}\right. \\
& \left.-\frac{1}{4} F_{a \alpha \beta} F_{a}^{\alpha \beta}\right\},
\end{aligned}
$$

where $\psi_{j}(u)$ is a four-component Dirac field, $d^{2} u \sqrt{-g}$ is the invariant volume element, $T^{a}=\lambda^{a} / 2$ are the eight matrix generators of $S U(3)$ color and $B_{a \alpha}$ are the color gauge fields. From this action, in the nonrelativistic limit, one obtains the effective Hamiltonian [129] composed of three terms (the quark, the string and the Coulomb): 


$$
\begin{aligned}
\mathcal{H}= & \mathcal{H}_{q}+\mathcal{H}_{s}+\mathcal{H}_{c} \\
= & \int d \sigma \chi^{+}\left(M \beta-i \alpha_{1} \partial_{1}\right) \chi+\int d \sigma \chi^{+} \beta \chi \frac{M v^{2}}{2} \\
& +\frac{e^{2}}{2} \int d \sigma d \sigma^{\prime} \chi^{+}(\sigma) T^{a} \chi(\sigma) G\left(\sigma, \sigma^{\prime}\right) \chi^{+}\left(\sigma^{\prime}\right) T^{a} \chi\left(\sigma^{\prime}\right),
\end{aligned}
$$

which, in absence of vibrations and after quantization of the rotational modes, leads to the following Schrödinger equation for the meson bound-states in the center-of-mass frame

$$
\left[2 M-\frac{1}{M} \frac{\partial^{2}}{\partial r^{2}}+k r-\frac{l(l+1)}{M r^{2}}\right] \psi(r)=E \psi(r) .
$$

The coupled equations that describe the dynamics of the string and the quark sectors are very non-linear so that there is no hope of solving them completely. Then, to introduce the vibrational modes, we use the following approximation scheme. First, we solve the string Hamiltonian (via de Bohr-Oppenheimer method) to obtain the vibrational energies as a function of the interquark distance. These are then inserted into the meson equation as an effective potential, $V_{n}(r)$.

Assuming the quark mass to be very heavy so that the ends of the string are fixed, the vibrational potential energy can be estimated using the Bohr-Sommerfeld quantization to be [129]

$$
V_{n}(r)=\sigma r\left\{1+\frac{2 n \pi}{\sigma\left[(r-2 d)^{2}+4 d^{2}\right]}\right\}^{1 / 2},
$$

where $d$ is the correction due to the finite quark mass

$$
d\left(m_{Q}, r, \sigma, n\right)=\frac{\sigma r^{2} \alpha_{n}}{4\left(2 m_{Q}+\sigma r \alpha_{n}\right)},
$$

being $\alpha_{n}$ a parameter related with the shape of the vibrating string [129], and can take the values $1 \leq \alpha_{n}^{2} \leq 2$. For $n=0$, $V_{n}(r)$ reduces to the naive $Q \bar{Q}$ one.

In our quark model, the central part of the confining potential has the following form

$$
V_{\mathrm{CON}}^{\mathrm{C}}(r)=\frac{16}{3}\left[a_{c}\left(1-e^{-\mu_{c} r}\right)-\Delta\right],
$$

and can be written as

$$
V_{\mathrm{CON}}^{\mathrm{C}}(r)=\sigma(r) r+\text { cte, }
$$

where

$$
\begin{aligned}
\sigma(r) & =\frac{16}{3} a_{c}\left(\frac{1-e^{-\mu_{c} r}}{r}\right), \\
\text { cte } & =-\frac{16}{3} \Delta .
\end{aligned}
$$

This means that our effective string tension, $\sigma(r)$, is not a constant but depends on the interquark distance, $r$. In fact, it decreases with respect to $r$ until it reaches the string breaking region.

Following the ideas of Ref. [130], the potential for hybrid mesons derived from our constituent quark model has the following expression [82]

$V_{\mathrm{hyb}}(r)=V_{\mathrm{OGE}}^{\mathrm{C}}(r)+V_{\mathrm{CON}}^{\mathrm{C}}(r)+\left[V_{n}(r)-\sigma(r) r\right]$,

where we have not taken into account the spin-dependent terms. $V_{\mathrm{OGE}}^{\mathrm{C}}(r)+V_{\mathrm{CON}}^{\mathrm{C}}(r)$ is the naive quark-antiquark potential and $V_{n}(r)$ is the vibrational one. We must subtract the term $\sigma(r) r$ because it appears twice, one in $V_{\mathrm{CON}}^{\mathrm{C}}(r)$ and the other one in $V_{n}(r)$. This potential does not include new model parameters and depends only on those coming from the original quark model. In such sense, the calculation of the hybrid states is parameter-free. More explicitly, our different contributions are

$$
\begin{aligned}
V_{\mathrm{OGE}}^{\mathrm{C}}(r) & =-\frac{4 \alpha_{s}}{3 r}, \\
V_{\mathrm{CON}}^{\mathrm{C}}(r) & =\frac{16}{3}\left[a_{c}\left(1-e^{-\mu_{c} r}\right)-\Delta\right], \\
V_{n}(r) & =\sigma(r) r\left\{1+\frac{2 n \pi}{\sigma(r)\left[(r-2 d)^{2}+4 d^{2}\right]}\right\}^{1 / 2},
\end{aligned}
$$

where

TABLE XVII. Hybrid meson masses, in MeV, calculated in the $b \bar{b}$ sector. The variation of the parameter $\alpha_{n}$ which range between $1<\alpha_{n}<\sqrt{2}$ modifies the energy as much as $30 \mathrm{MeV}$, we have taken $\alpha_{n}=\sqrt{1.5}$.

\begin{tabular}{lccc}
\hline \hline $\mathrm{K}$ & $L=0$ & $L=1$ & $L=2$ \\
\hline 1 & 10571 & 10785 & 10921 \\
2 & 10857 & 10999 & 11108 \\
3 & 11063 & 11175 & 11267 \\
4 & 11232 & 11325 & 11402 \\
5 & 11374 & 11452 & 11519 \\
6 & 11496 & 11562 & 11619 \\
7 & 11600 & 11657 & 11706 \\
8 & 11690 & 11738 & 11780 \\
9 & 11766 & 11807 & 11843 \\
10 & 11831 & 11866 & 11895 \\
11 & 11885 & 11913 & $\cdots$ \\
12 & 11927 & $\cdots$ & $\cdots$ \\
Threshold $=11943 \mathrm{MeV}$ & & \\
\hline \hline
\end{tabular}




$$
d\left(m_{Q}, r, \sigma, n\right)=\frac{\sigma(r) r^{2} \alpha_{n}}{4\left(2 m_{Q}+\sigma(r) r \alpha_{n}\right)} .
$$

An important feature of our hybrid model is that, just like the naive quark model, the hybrid potential has a threshold from which no more states can be found and so we have a finite number of hybrid states in the spectrum. Hybrid meson masses calculated in the bottomonium sector using our model are shown in Table XVII.
[1] S. Herb, D. Hom, L. Lederman, J. Sens, H. Snyder et al., Phys. Rev. Lett. 39, 252 (1977).

[2] W. R. Innes, J. Appel, B. Brown, C. Brown, K. Ueno et al., Phys. Rev. Lett. 39, 1240 (1977).

[3] K. Han, T. Bohringer, P. Franzini, G. Mageras, D. Peterson et al., Phys. Rev. Lett. 49, 1612 (1982).

[4] G. Eigen, G. Blanar, H. Dietl, E. Lorenz, F. Pauss et al., Phys. Rev. Lett. 49, 1616 (1982).

[5] C. Klopfenstein, J. Horstkotte, J. Lee-Franzini, R. D. Schamberger, M. Sivertz et al., Phys. Rev. Lett. 51, 160 (1983).

[6] F. Pauss, H. Dietl, G. Eigen, E. Lorenz, G. Mageras et al., Phys. Lett. B 130, 439 (1983).

[7] D. Besson et al. (CLEO), Phys. Rev. Lett. 54, 381 (1985).

[8] D. Lovelock, J. Horstkotte, C. Klopfenstein, J. LeeFranzini, L. Romero et al., Phys. Rev. Lett. 54, 377 (1985).

[9] B. Aubert et al. (BABAR), Phys. Rev. Lett. 101, 071801 (2008).

[10] G. Bonvicini et al. (CLEO Collaboration), Phys. Rev. D 81, 031104 (2010).

[11] R. Mizuk et al. (Belle), Phys. Rev. Lett. 109, 232002 (2012).

[12] J. Lees et al. (BABAR), Phys. Rev. D 84, 072002 (2011).

[13] S. Dobbs, Z. Metreveli, K. K. Seth, A. Tomaradze, and T. Xiao, Phys. Rev. Lett. 109, 082001 (2012).

[14] S. Sandilya et al. (Belle), Phys. Rev. Lett. 111, 112001 (2013).

[15] G. Bonvicini et al. (CLEO Collaboration), Phys. Rev. D 70, 032001 (2004).

[16] P. del Amo Sanchez et al. (BABAR), Phys. Rev. D 82, 111102 (2010).

[17] J. Lees et al. (BABAR), Phys. Rev. D 84, 091101 (2011).

[18] I. Adachi et al. (Belle Collaboration), Phys. Rev. Lett. 108, 032001 (2012).

[19] G. Aad et al. (ATLAS Collaboration), Phys. Rev. Lett. 108, 152001 (2012).

[20] V. M. Abazov et al. (D0 Collaboration), Phys. Rev. D 86, 031103 (2012).

[21] R. Aaij et al. (LHCb), J. High Energy Phys. 10 (2014) 88.

[22] K. A. Olive et al. (Particle Data Group), Chin. Phys. C 38, 090001 (2014).

[23] A. Bondar et al. (Belle), Phys. Rev. Lett. 108, 122001 (2012).

[24] D. Bugg, Europhys. Lett. 96, 11002 (2011).

[25] I. Danilkin, V. Orlovsky, and Y. Simonov, Phys. Rev. D 85, 034012 (2012).
[26] C.-Y. Cui, Y.-L. Liu, and M.-Q. Huang, Phys. Rev. D 85, 074014 (2012).

[27] T. Guo, L. Cao, M.-Z. Zhou, and H. Chen, arXiv:1106.2284.

[28] M. Gómez-Rocha, T. Hilger, and A. Krassnigg, Few Body Syst. 56, 475 (2015).

[29] T. Hilger, C. Popovici, M. Gomez-Rocha, and A. Krassnigg, Phys. Rev. D 91, 034013 (2015).

[30] C. S. Fischer, S. Kubrak, and R. Williams, Eur. Phys. J. A 51, 10 (2015).

[31] W. Caswell and G. Lepage, Phys. Lett. B 167, 437 (1986).

[32] G. T. Bodwin, E. Braaten, and G. P. Lepage, Phys. Rev. D 51, 1125 (1995); 55, 5853(E) (1997).

[33] A. Pineda and J. Soto, Nucl. Phys. B, Proc. Suppl. 64, 428 (1998).

[34] N. Brambilla, A. Pineda, J. Soto, and A. Vairo, Nucl. Phys. B566, 275 (2000).

[35] N. Brambilla, A. Pineda, J. Soto, and A. Vairo, Rev. Mod. Phys. 77, 1423 (2005).

[36] A. Pineda, Prog. Part. Nucl. Phys. 67, 735 (2012).

[37] J. J. Dudek, R. G. Edwards, N. Mathur, and D. G. Richards, Phys. Rev. D 77, 034501 (2008).

[38] N. Brambilla, S. Eidelman, B. Heltsley, R. Vogt, G. Bodwin et al., Eur. Phys. J. C 71, 1534 (2011).

[39] A. Gray, I. Allison, C. T. H. Davies, E. Gulez, G. P. Lepage, J. Shigemitsu, and M. Wingate, Phys. Rev. D 72, 094507 (2005).

[40] S. Meinel, Phys. Rev. D 79, 094501 (2009).

[41] T. Burch, C. DeTar, M. Di Pierro, A. X. El-Khadra, E. D. Freeland, S. Gottlieb, A. S. Kronfeld, L. Levkova, P. B. Mackenzie, and J. N. Simone, Phys. Rev. D 81, 034508 (2010).

[42] S. Meinel, Phys. Rev. D 82, 114502 (2010).

[43] R. Dowdall et al. (HPQCD), Phys. Rev. D 85, 054509 (2012).

[44] R. Lewis and R. Woloshyn, Phys. Rev. D 85, 114509 (2012).

[45] M. Wurtz, R. Lewis, and R. M. Woloshyn, Phys. Rev. D 92, 054504 (2015).

[46] E. Eichten, K. Gottfried, T. Kinoshita, K. Lane, and T.-M. Yan, Phys. Rev. D 17, 3090 (1978).

[47] E. Eichten, K. Gottfried, T. Kinoshita, K. Lane, and T.-M. Yan, Phys. Rev. D 21, 203 (1980).

[48] S. N. Gupta, S. F. Radford, and W. W. Repko, Phys. Rev. D 26, 3305 (1982).

[49] S. N. Gupta, S. F. Radford, and W. W. Repko, Phys. Rev. D 28, 1716 (1983). 
[50] S. N. Gupta, S. F. Radford, and W. W. Repko, Phys. Rev. D 30, 2424 (1984).

[51] S. N. Gupta, S. F. Radford, and W. W. Repko, Phys. Rev. D 31, 160 (1985).

[52] W. Kwong, P. B. Mackenzie, R. Rosenfeld, and J. L. Rosner, Phys. Rev. D 37, 3210 (1988).

[53] W. Kwong and J. L. Rosner, Phys. Rev. D 38, 279 (1988).

[54] T. Barnes, F. Close, P. Page, and E. Swanson, Phys. Rev. D 55, 4157 (1997).

[55] D. Ebert, R. Faustov, and V. Galkin, Phys. Rev. D 67, 014027 (2003).

[56] S. F. Radford and W. W. Repko, Phys. Rev. D 75, 074031 (2007).

[57] E. Eichten, S. Godfrey, H. Mahlke, and J. L. Rosner, Rev. Mod. Phys. 80, 1161 (2008).

[58] I. Danilkin and Y. Simonov, Phys. Rev. D 81, 074027 (2010).

[59] J. Ferretti and E. Santopinto, Phys. Rev. D 90, 094022 (2014).

[60] S. Godfrey and K. Moats, Phys. Rev. D 92, 054034 (2015).

[61] L. Micu, Nucl. Phys. B10, 521 (1969).

[62] A. L. Yaouanc, L. Oliver, O. Pene, and J. Raynal, Phys. Rev. D 8, 2223 (1973).

[63] A. L. Yaouanc, L. Oliver, O. Pene, and J.-C. Raynal, Phys. Rev. D 9, 1415 (1974).

[64] S. Capstick and N. Isgur, Phys. Rev. D 34, 2809 (1986).

[65] S. Capstick and W. Roberts, Phys. Rev. D 49, 4570 (1994).

[66] P. R. Page, Nucl. Phys. B446, 189 (1995).

[67] E. Ackleh, T. Barnes, and E. Swanson, Phys. Rev. D 54, 6811 (1996).

[68] J. Vijande, F. Fernandez, and A. Valcarce, J. Phys. G 31, 481 (2005).

[69] J. Segovia, D. Entem, and F. Fernandez, Phys. Lett. B 662 , 33 (2008).

[70] J. Segovia, A. Yasser, D. Entem, and F. Fernandez, Phys. Rev. D 78, 114033 (2008).

[71] B. A. Kniehl, A. A. Penin, A. Pineda, V. A. Smirnov, and M. Steinhauser, Phys. Rev. Lett. 92, 242001 (2004).

[72] J. Segovia, D. R. Entem, F. Fernandez, and E. R. Arriola, Phys. Rev. D 85, 074001 (2012).

[73] J. Segovia, D. R. Entem, F. Fernandez, and E. R. Arriola, Phys. Rev. D 86, 094027 (2012).

[74] S. Godfrey and J. L. Rosner, Phys. Rev. D 66, 014012 (2002).

[75] N. Brambilla, Y. Jia, and A. Vairo, Phys. Rev. D 73, 054005 (2006).

[76] A. Pineda and J. Segovia, Phys. Rev. D 87, 074024 (2013).

[77] B. Aubert et al. (BABAR), Phys. Rev. Lett. 103, 161801 (2009).

[78] C. Hughes, R. J. Dowdall, C. T. H. Davies, R. R. Horgan, G. von Hippel, and M. Wingate, Phys. Rev. D 92, 094501 (2015).

[79] J. Lees et al. (BABAR), Phys. Rev. D 90, 112010 (2014).

[80] B. Aubert et al. (BABAR), Phys. Rev. Lett. 102, 012001 (2009).

[81] K.-F. Chen et al. (Belle), Phys. Rev. D 82, 091106 (2010).

[82] J. Segovia, D. R. Entem, and F. Fernandez, Phys. Rev. D 91, 014002 (2015).

[83] J. Segovia, F. Fernandez, and D. R. Entem, Few Body Syst. 57, 275 (2016).
[84] A. Ali, C. Hambrock, and M. J. Aslam, Phys. Rev. Lett. 104, 162001 (2010).

[85] J. Segovia, D. Entem, and F. Fernandez, Phys. Lett. B 715 , 322 (2012).

[86] E. Eichten and F. Feinberg, Phys. Rev. D 23, 2724 (1981).

[87] S. Gupta, S. Radford, and W. Repko, Phys. Rev. Lett. 55, 3006 (1985).

[88] P. Moxhay and J. L. Rosner, Phys. Rev. D 28, 1132 (1983).

[89] A. Badalian, B. Ioffe, and A. V. Smilga, Nucl. Phys. B281, 85 (1987).

[90] A. Valcarce, H. Garcilazo, F. Fernandez, and P. Gonzalez, Rep. Prog. Phys. 68, 965 (2005).

[91] J. Segovia, D. Entem, F. Fernandez, and E. Hernandez, Int. J. Mod. Phys. E 22, 1330026 (2013).

[92] F. Fernandez, A. Valcarce, P. Gonzalez, and V. Vento, Phys. Lett. B 287, 35 (1992).

[93] H. Garcilazo, A. Valcarce, and F. Fernandez, Phys. Rev. C 63, 035207 (2001); 64, 058201 (2001).

[94] J. Vijande, H. Garcilazo, A. Valcarce, and F. Fernandez, Phys. Rev. D 70, 054022 (2004).

[95] J. Segovia, A. Yasser, D. Entem, and F. Fernandez, Phys. Rev. D 80, 054017 (2009).

[96] J. Segovia, D. R. Entem, and F. Fernandez, Phys. Rev. D 91, 094020 (2015).

[97] J. Segovia, D. Entem, and F. Fernandez, Phys. Rev. D 83, 114018 (2011).

[98] J. Segovia, D. Entem, and F. Fernandez, Nucl. Phys. A915, 125 (2013).

[99] J. Segovia, C. Albertus, D. R. Entem, F. Fernández, E. Hernández, and M. A. Pérez-García, Phys. Rev. D 84, 094029 (2011).

[100] J. Segovia, C. Albertus, E. Hernandez, F. Fernandez, and D. Entem, Phys. Rev. D 86, 014010 (2012).

[101] J. Segovia, E. Hernandez, F. Fernandez, and D. Entem, Phys. Rev. D 87, 114009 (2013).

[102] E. Hiyama, Y. Kino, and M. Kamimura, Prog. Part. Nucl. Phys. 51, 223 (2003).

[103] N. Brambilla, P. Pietrulewicz, and A. Vairo, Phys. Rev. D 85, 094005 (2012).

[104] R. Barbieri, R. Gatto, R. Kogerler, and Z. Kunszt, Phys. Lett. B 57, 455 (1975).

[105] V. A. Novikov, L. B. Okun, M. A. Shifman, A. I. Vainshtein, M. B. Voloshin, and V. I. Zakharov, Phys. Rep. 41, 1 (1978).

[106] A. Bradley and A. Khare, Z. Phys. C 8, 131 (1981).

[107] E. S. Ackleh and T. Barnes, Phys. Rev. D 45, 232 (1992).

[108] G. Belanger and P. Moxhay, Phys. Lett. B 199, 575 (1987).

[109] N. Brambilla, D. Eiras, A. Pineda, J. Soto, and A. Vairo, Phys. Rev. Lett. 88, 012003 (2001).

[110] N. Brambilla, D. Eiras, A. Pineda, J. Soto, and A. Vairo, Phys. Rev. D 67, 034018 (2003).

[111] A. Vairo, Mod. Phys. Lett. A 19, 253 (2004).

[112] J. Segovia, Ph.D. thesis, Universidad de Salamanca, 2012, http://inspirehep.net/record/1316438.

[113] S. Okubo, Phys. Lett. 5, 165 (1963)

[114] G. Zweig, Report No. CERN-TH-412, NP-8419 (1964).

[115] J. Iizuka, Prog. Theor. Phys. Suppl. 37, 21 (1966).

[116] K. Gottfried, Phys. Rev. Lett. 40, 598 (1978).

[117] G. Bhanot, W. Fischler, and S. Rudaz, Nucl. Phys. B155, 208 (1979). 
[118] M. E. Peskin, Nucl. Phys. B156, 365 (1979).

[119] G. Bhanot and M. E. Peskin, Nucl. Phys. B156, 391 (1979).

[120] M. Voloshin, Nucl. Phys. B154, 365 (1979).

[121] M. B. Voloshin and V. I. Zakharov, Phys. Rev. Lett. 45, 688 (1980).

[122] T.-M. Yan, Phys. Rev. D 22, 1652 (1980).

[123] Y.-P. Kuang and T.-M. Yan, Phys. Rev. D 24, 2874 (1981).

[124] Y.-P. Kuang, Front. Phys. China 1, 19 (2006).

[125] L. S. Brown and R. N. Cahn, Phys. Rev.. Lett. 35, 1 (1975).
[126] L. Liu, G. Moir, M. Peardon, S. M. Ryan, C. E. Thomas, P. Vilaseca, J. J. Dudek, R. G. Edwards, B. Joo, and D. G. Richards (Hadron Spectrum), J. High Energy Phys. 07 (2012) 126.

[127] M. Berwein, N. Brambilla, J. Tarrus-Castella, and A. Vairo, Phys. Rev. D 92, 114019 (2015).

[128] S. Tye, Phys. Rev. D 13, 3416 (1976).

[129] R. Giles and S. Tye, Phys. Rev. D 16, 1079 (1977).

[130] W. Buchmuller and S. Tye, Phys. Rev. Lett. 44, 850 (1980). 